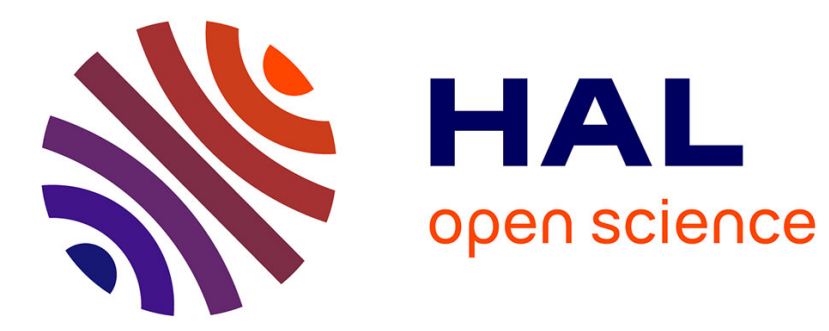

\title{
Plane strain dynamics of elastic solids with intrinsic boundary elasticity, with application to surface wave propagation
}

\author{
R. W. Ogden, D. J. Steigmann
}

\section{To cite this version:}

R. W. Ogden, D. J. Steigmann. Plane strain dynamics of elastic solids with intrinsic boundary elasticity, with application to surface wave propagation. Journal of the Mechanics and Physics of Solids, 2002, 50 (9), pp.1869-1896. 10.1016/S0022-5096(02)00006-6 . hal-01297748

HAL Id: hal-01297748

https://hal.science/hal-01297748

Submitted on 4 Apr 2016

HAL is a multi-disciplinary open access archive for the deposit and dissemination of scientific research documents, whether they are published or not. The documents may come from teaching and research institutions in France or abroad, or from public or private research centers.
L'archive ouverte pluridisciplinaire HAL, est destinée au dépôt et à la diffusion de documents scientifiques de niveau recherche, publiés ou non, émanant des établissements d'enseignement et de recherche français ou étrangers, des laboratoires publics ou privés. 


\title{
Plane strain dynamics of elastic solids with intrinsic boundary elasticity, with application to surface wave propagation
}

\author{
R.W. Ogden ${ }^{\mathrm{a}, *}$, D.J. Steigmann ${ }^{\mathrm{b}}$ \\ aepartment of Mathematics, Unitersity of Glasyow; Unitersity Gardens, \\ Glasgow GI2 SQW, UK \\ ${ }^{b}$ Deparment of Mechanical Engineering. Unitersity of California at Berkeley. Berkeley. \\ CA 94720, USA
}

\begin{abstract}
In this paper, in a development of the static theory derived by Steigmann and Ogden (Proc. Roy. Soc. London A 453 (1997) 853), we establish the equations of motion for a non-linearly elastic body in plane strain with an elastic surface coating on part or all of its boundary. The equations of (linearized) incremental motions superposed on a finite static deformation are then obtained and applied to the problem of (time-harmonic) surface wave propagation on a pre-stressed incompressible isotropic elastic half-space with a thin coating on its plane boundary. The secular equation for (dispersive) wave speeds is then obtained in respect of a general form of incompressible isotropic elastic strain-energy function for the bulk material and a general energy function for the coating material. Specialization of the form of strain-energy function enables the secular equation to be cast as a quartic equation and we therefore focus on this for illustrative purposes. An explicit form for the secular equation is thereby obtained. This involves a number of material parameters, including residual stress and moment in the properties of the coating. It is shown how this equation relates to previous work on waves in a half-space with an overlying thin layer set in the classical theory of isotropic elasticity and, in particular, the significant effect of omission of the rotatory inertia term, even at small wave numbers, is emphasized. Corresponding results for a membrane-type coating, for which the bending moment, inertia and residual moment terms are absent, are also obtained. Asymptotic formulas for the wave speed at large wave number (high frequency) are derived and it is shown how these results influence the character of the wave speed throughout the range of wave number values. A bifurcation criterion is obtained from the secular equation by setting the wave speed to zero, thereby generalizing the bifurcation results of Steigmann and Ogden (Proc. Roy. Soc. London A 453 (1997) 853) to
\end{abstract}

*Corresponding author. Tel.: +44-141-330-4550; fax: +44-141-330-4111.

E-mail addresses: rwo@ maths.gla.ac.uk (R.W. Ogden), steigman@me.berkeley.edu (D.J. Steigmann). 
the situation in which residual stress and moment are present in the coating. Numerical results which show the dependence of the wave speed on the various material parameters and the finite deformation are then described graphically. In particular, features which differ from those arising in the classical theory are highlighted. (C) 2002 Elsevier Science Ltd. All rights reserved.

Keywords: A. Elastic solids; Non-linear elasticity; Surface stress; Dynamics; C. Wave propagation

\section{Introduction}

In a recent paper (Steigmann and Ogden, 1997a), henceforth referred to as (I), the authors have developed a static non-linear plane strain theory of an elastic solid coated with a thin film of elastic material that has both extensional and flexural resistance. Applications of the theory were examined in (I), Ogden et al. $(1997,1998)$ and Dryburgh and Ogden (1999), and further theory was provided in Steigmann and Ogden (1997b). The corresponding three-dimensional theory is described in Steigmann and Ogden (1999).

In the model for the surface coating, which extends that developed by Gurtin and Murdoch $(1975,1979)$ for membrane surfaces to allow for the inclusion of flexural stiffness, the mechanical response of the coating is determined by the metric and curvature of the surface. Motivation for inclusion of flexural stiffness in the considered model is discussed in detail in (I) and in Steigmann and Ogden (1999), but it is appropriate to highlight certain key points here. Firstly, the significance of flexural stiffness in respect of the mechanical stability of coatings produced by thin-film deposition processes has been elucidated by Gille and Rau (1984) and Yu et al. (1991). In the absence of flexural stiffness the coating cannot support compressive stresses. This fact effectively rules out the pure membrane theory of Gurtin and Murdoch as a model for equilibrium states that support compression in the surface film. Indeed, according to the energy criterion of elastic stability, such states cannot be maintained in stable equilibrium, as was proved in (I). But, experiments indicate the presence of significant compressive stresses in the surface of certain thin-film/substrate systems (see, for example, Krulevitch et al. (1992), Kuba and Sedláček (1990) and the review by Nix (1989)). Thus, in order to accommodate such compressive stresses in equilibrium and to analyse flexural wave propagation in compressed states it is essential to use a model which incorporates flexural stiffness.

In the context of plane strain, $\mathrm{Wu}$ (1996) presented an interesting extension of the Gurtin-Murdoch theory to account for the effects of material accretion at boundaries. His kinematical formulas are similar to those used here. He suppressed the dependence of the strain energy on local curvature, an effect that we include, while in the present work the boundary film is considered to be a material surface in all configurations and thus the effects of accretion considered by $\mathrm{Wu}$ are not included. See also $\mathrm{Wu}$ et al. (1998) and references to related work in these two papers.

In the present paper we extend the (plane strain) theory to allow for the dynamics of a substrate-coating structure, including the effects of the rotatory inertia of the coating. The theory is then applied to a prototype problem in which infinitesimal surface 
waves of Rayleigh type are examined for a statically pre-stressed half-space of incompressible isotropic elastic material coated with a thin film on its plane boundary. This extends to the dynamic context the results obtained in (I) relating to the quasi-static bifurcation of a pre-stressed half-space. For classical isotropic elasticity, surface waves in a membrane-coated half-space with a residual surface tension were discussed by Murdoch (1976), and his results may be recovered by appropriate specialization of the theory discussed here.

Our results are discussed in relation to those for a thin, but finite thickness, layer of material on a half-space (see, for example, Achenbach and Keshava (1967), Tiersten (1969) and Farnell and Adler (1972) in the classical context and Ogden and Sotiropoulos (1995) for the case of pre-stressed materials) and to those with the layer modelled using plate theory (Achenbach and Keshava, 1967; Tiersten, 1969). It is shown, in particular, that rotatory inertia, which is sometimes neglected in the classical theory (Tiersten, 1969), can have a significant influence on the surface wave behaviour even at relatively small values of the wave number.

In Section 2, we summarize the basic equations for the bulk solid/film combination and derive the equations governing the motion of the film. The role of the rotatory inertia term is discussed in some detail. We also derive the equations governing the coupling of mechanical power between bulk and film material and the associated energy flux. In Section 3, we obtain the (linearized) incremental equations governing small motions superimposed on a finite static deformation. The equations are then applied, in Section 4, to the problem of infinitesimal surface waves propagating in a homogeneously pure strained incompressible isotropic elastic half-space with an elastic coating on its plane boundary. The two equations governing the motion of the film provide boundary conditions for the displacement in the bulk solid which, since the material is incompressible, is governed by a single scalar equation.

Surface wave solutions of harmonic type are used to obtain, on application of the boundary conditions, the secular equation for the speed of surface waves. Unlike in the situation for an uncoated half-space (Dowaikh and Ogden, 1990), the waves here are dispersive and the wave speed depends on the deformation in the bulk material, the properties of the bulk and film materials, the wave number, the relative densities of the two materials and the local moment of inertia of the film cross-section. An explicit form of the secular equation is given in respect of an arbitrary incompressible, isotropic elastic substrate material and an arbitrary film material whose properties are described by a strain energy per unit reference length that depends only on the stretch and curvature of the film, as in (I). Additionally, we include in the properties of the film material both residual stress and residual moment. For a special form of energy function for the bulk material the secular equation reduces to a quartic, which contrasts with the cubic obtained for the uncoated case. Material properties for the coating material and a number of specializations of the secular equation are discussed in Section 5. In particular, the secular equation derived by Dowaikh and Ogden (1990) for an uncoated half-space is recovered. Furthermore, the specialization of the secular equation for the undeformed configuration, with residual stress and moment incorporated, is given, and this, when the flexural rigidity is omitted, can be shown to be equivalent to an equation obtained by Murdoch (1976) for a membrane film. A different specialization, again for 
the undeformed situation but with residual stress and moment excluded and rotatory inertia included, enables the results to be compared with those of Tiersten (1969).

In Section 6, asymptotic results for large wave number are derived and it is shown that these have a significant influence on the character of the wave speed throughout the range of wave numbers. It is also shown that when the wave speed is set to zero in the secular equation a bifurcation equation is obtained which generalizes that given in (I) for incompressible materials to the case in which residual stress and moment are included in the coating. Finally, in Section 7, numerical solutions of the secular equation are presented in graphical form to illustrate the dependence of the wave speed on the various material and geometrical parameters.

\section{Basic equations}

\subsection{Kinematics}

We consider plane motions of a (prismatic) body whose plane section is identified in its reference configuration with a two-dimensional region $\Omega$. The motion is described by a mapping from $\Omega$ to the two-dimensional region $\hat{\Omega}$. A material particle is labelled by its position vector $\mathbf{X}$ in $\Omega$, and its position in $\hat{\Omega}$ is denoted by $\mathbf{x}$. The motion is defined by the mapping $\chi$ such that

$$
\mathbf{x}=\chi(\mathbf{X}, t), \quad \mathbf{X} \in \Omega, t \in \mathscr{I},
$$

where $t$ is the time and $\mathscr{I}$ some appropriate time interval. For each $t \in \mathscr{I}, \chi$ is invertible and is assumed to possess regularity properties necessary for the ensuing analysis.

The deformation gradient tensor $\mathbf{A}$ is given by

$$
\mathbf{A}=\operatorname{Grad} \mathbf{x},
$$

where Grad is the (two-dimensional) gradient operator on $\Omega$. Let $\left\{\mathbf{e}_{1}, \mathbf{e}_{2}\right\}$ be a fixed orthonormal basis in the plane under consideration and let $x_{i}=\mathbf{x} \cdot \mathbf{e}_{i}, X_{\alpha}=\mathbf{X} \cdot \mathbf{e}_{\alpha}$, with $i, \alpha \in\{1,2\}$, denote the Cartesian coordinates of a particle in $\hat{\Omega}$ and $\Omega$, respectively. Then, in component form, Eq. (2) may be written as

$$
\mathbf{A}=A_{i \alpha} \mathbf{e}_{i} \otimes \mathbf{e}_{\alpha}, \quad A_{i \alpha}=x_{i, \alpha} \equiv \frac{\partial x_{i}}{\partial X_{\alpha}} .
$$

We also note the (two-dimensional) polar decomposition

$$
\mathbf{A}=\mathbf{R} \mathbf{U},
$$

where $\mathbf{R}$ is proper orthogonal and $\mathbf{U}$ is positive definite and symmetric. The right stretch tensor $\mathbf{U}$ has the spectral decomposition

$$
\mathbf{U}=\lambda_{1} \mathbf{u}^{(1)} \otimes \mathbf{u}^{(1)}+\lambda_{2} \mathbf{u}^{(2)} \otimes \mathbf{u}^{(2)},
$$

where $\lambda_{1}$ and $\lambda_{2}$ are the principal stretches of the deformation and $\left\{\mathbf{u}^{(1)}, \mathbf{u}^{(2)}\right\}$ the associated (orthonormal) principal directions of $\mathbf{U}$. Since we are considering plane 
deformations it is implicit that the stretch $\lambda_{3}$ normal to the considered plane is set to unity. Then, for an isochoric deformation, we have

$$
\lambda_{1} \lambda_{2}=1 \text {. }
$$

Let $S$ be the arclength parameter describing the boundary $\partial \Omega$ of $\Omega$ in the usual sense and let $\mathbf{N}$ be the rightward unit normal to $\partial \Omega$. We write $\mathbf{X}(S)$ as the parametrization of points on $\partial \Omega$, as discussed in (I).

Let a subset $P$ of $\partial \Omega$ be coated with a thin elastic film that deforms as a material curve. The image of $P$ on $\partial \hat{\Omega}$, the boundary of $\hat{\Omega}$, is denoted by $\hat{P}$, and we write

$$
\mathbf{r}(S, t)=\chi(\mathbf{X}(S), t)
$$

for those values of $S$ for which $\mathbf{X}(S)$ is on $P$. The unit tangent to $P$ is $\mathbf{T}(S)=\mathbf{X}^{\prime}(S)$, where the prime indicates differentiation with respect to $S$.

Differentiation of Eq. (7) with respect to $S$ yields

$$
\mathbf{r}^{\prime}(S, t)=\mathbf{A}(\mathbf{X}(S), t) \mathbf{T}(S) \equiv \lambda(S, t) \tau(S, t),
$$

where

$$
\lambda(S, t)=\left|\mathbf{A}(\mathbf{X}(S), t) \mathbf{X}^{\prime}(S)\right|
$$

is the stretch of $P$ induced by the motion $\chi, \tau(S, t)$ is the unit tangent to $\hat{P}$ at the point with arclength station $S$ on $P$ at time $t$, and the prime represents $\partial / \partial S$. Let $s$, which depends on $S$ and $t$, be the arclength parameter for $\hat{P}$; we then have $s^{\prime}(S, t)=\lambda(S, t)$.

Let $\theta(S, t)$ be the angle that the tangent $\chi$ to $\hat{P}$ makes with the axis $\mathbf{e}_{1}$ measured in the counterclockwise sense. Then, we have

$$
\tau(S, t)=\cos \theta(S, t) \mathbf{e}_{1}+\sin \theta(S, t) \mathbf{e}_{2} .
$$

We denote by $\boldsymbol{v}(S, t)=\mathbf{k} \times \tau(S, t)$ the leftward unit normal to $\hat{P}$, where $\mathbf{k}=\mathbf{e}_{1} \times \mathbf{e}_{2}$ is the unit normal to the plane of $\Omega$ and $\hat{\Omega}$. Then,

$$
\tau^{\prime}(S, t)=\kappa(S, t) v(S, t), \quad \kappa(S, t) \equiv \theta^{\prime}(S, t),
$$

with the curvature of $\hat{P}$ being $\lambda^{-1} \kappa$.

\subsection{Constitutive laws}

Let $W(\mathbf{A})$ be the strain energy of the bulk material per unit area of $\Omega$. Since the material is assumed to be incompressible, with the constraint

$$
\operatorname{det} \mathbf{A}=1 \quad \text { in } \Omega
$$

holding, the (plane) nominal stress tensor $\mathbf{S}$ is given by

$$
\mathbf{S}=\frac{\partial W}{\partial \mathbf{A}}-p \mathbf{A}^{-1}
$$

$p$ being a Lagrange multiplier arising from constraint (12). There will also be a stress normal to the considered plane necessary to maintain the plane strain condition, but we do not require an explicit expression for it here. 
For an isotropic material the corresponding Cauchy stress $\boldsymbol{\sigma}=\mathbf{A S}$ has principal components

$$
\sigma_{1}=\lambda_{1} \frac{\partial W}{\partial \lambda_{1}}-p, \quad \sigma_{2}=\lambda_{2} \frac{\partial W}{\partial \lambda_{2}}-p,
$$

where $W\left(\lambda_{1}, \lambda_{2}\right)=W\left(\lambda_{2}, \lambda_{1}\right)$, with $\lambda_{1} \lambda_{2}=1$.

Following Steigmann and Ogden (1997a,b), we take the strain energy of the coating, per unit length of $P$, to depend only on $\lambda$ and $\kappa$. We write this as $U(\lambda, \kappa)$, and use the notations defined by

$$
F=\frac{\partial U}{\partial \lambda} \equiv U_{\lambda}, \quad M=\frac{\partial U}{\partial \kappa} \equiv U_{\kappa},
$$

which represent the tangential force and moment on $\hat{P}$, respectively. The force at a point on $\hat{P}$ consists of both the tangential component $F$ and a normal component and may be written as

$$
\mathbf{F}=F \tau+G \boldsymbol{v},
$$

as described in (I), where $G$, the transverse shear force on the film, is not determined by a constitutive equation (it is a Lagrange multiplier). In this model the response of the coating is determined solely by the geometry of the surface (in this plane strain situation actually the curve corresponding to the coating). An alternative approach, which we do not consider here, would be to assign an independent director field to the surface which would not be directly related to the film geometry but would require an additional constitutive law for $G$.

In Steigmann and Ogden (1997b) it was proved that a necessary condition for a (static) deformed configuration of the film-substrate structure to be an energy minimizer is that the Hessian matrix

$$
\left(\begin{array}{ll}
U_{\lambda \lambda} & U_{\lambda \kappa} \\
U_{\lambda \kappa} & U_{\kappa \kappa}
\end{array}\right)
$$

is positive semidefinite. Moreover, it was established in (I) that for a membrane film (with no bending stiffness) a necessary condition is that the force $F$ is non-compressive, that is

$$
U_{\lambda} \geqslant 0 \text {. }
$$

\subsection{Equations of motion}

In the absence of body forces the equation of motion of the bulk material in $\Omega$ may be written in the standard form

$$
\operatorname{Div} \mathbf{S}=\rho \mathbf{x}_{, t},
$$

where $\rho$ is the mass per unit area of $\Omega$, Div is the divergence operator in $\Omega$ and ${ }_{, t}$ signifies the material time derivative. Boundary conditions were discussed in (I) for static problems, and such conditions can be carried over to the present context as 
needed, but here we shall not consider conditions on $\partial \Omega$ other than on the part $P$, which are provided by the following derivation of the equations of motion of $P$.

First, we discuss the balance of linear momentum for the film. Consider an arbitrary section $\left[S_{1}, S_{2}\right] \subset P$, where $S$ measures arclength along $P$ and $S_{2}>S_{1}$. Let $\mathbf{F}_{1}$ and $\mathbf{F}_{2}$, respectively be the forces applied to the ends $S_{1}$ and $S_{2}$ of the section, where $\mathbf{F}_{2}=\mathbf{F}\left(S_{2}, t\right)$ and $\mathbf{F}(S, t)$, given by Eq. (16), is the force exerted by the material in $\left(S, S_{2}\right]$ on that in $\left[S_{1}, S\right]$. The balance of linear momentum for the film is assumed to have the form

$$
\mathbf{F}_{1}+\mathbf{F}_{2}+\mathbf{B}=\frac{\mathrm{d}}{\mathrm{d} t} \int_{S_{1}}^{S_{2}} \rho_{0} \mathbf{r}_{, t} \mathrm{~d} S,
$$

where $\rho_{0}$ is the mass density of the film per unit reference length and

$$
\mathbf{B}=-\int_{S_{1}}^{S_{2}} \mathbf{S}^{\mathrm{T}} \mathbf{N} \mathrm{d} S
$$

the force exerted by the substrate on the film, is equal and opposite to that transmitted to the substrate by the film.

If we assume that the integrands in Eqs. (20) and (21) are bounded, that $\mathbf{F}(S, t)$ is a continuous function of $S$, and then let the length of the interval approach zero, we obtain $\mathbf{F}\left(S_{1}, t\right)+\mathbf{F}_{1}=\mathbf{0}$.

This allows us to write Eq. (20) as

$$
\int_{S_{1}}^{S_{2}}\left(\mathbf{F}^{\prime}-\mathbf{S}^{\mathrm{T}} \mathbf{N}-\rho_{0} \mathbf{r}_{t t}\right) \mathrm{d} S=\mathbf{0}
$$

and the arbitrariness of the interval then leads to the local equation of motion

$$
\mathbf{F}^{\prime}=\mathbf{S}^{\mathrm{T}} \mathbf{N}+\rho_{0} \mathbf{r}_{, t} .
$$

We also introduce a moment-of-momentum balance for the film. This is assumed to have the form

$$
\begin{gathered}
\left(M_{1}+M_{2}\right) \mathbf{k}+\mathbf{r}_{1} \times \mathbf{F}_{1}+\mathbf{r}_{2} \times \mathbf{F}_{2}+\int_{S_{1}}^{S_{2}} \mathbf{r} \times\left(-\mathbf{S}^{\mathrm{T}} \mathbf{N}\right) \mathrm{d} S \\
=\frac{\mathrm{d}}{\mathrm{d} t} \int_{S_{1}}^{S_{2}} \rho_{0} \mathbf{r} \times \mathbf{r}_{, t} \mathrm{~d} S+\mathbf{k} \frac{\mathrm{d}}{\mathrm{d} t} \int_{S_{1}}^{S_{2}} I \theta_{, t} \mathrm{~d} S,
\end{gathered}
$$

where $M_{1}$ and $M_{2}$ are the moments applied to the ends of the interval, $\mathbf{k}=\tau \times \boldsymbol{v}$ is the fixed unit normal to the plane of motion, and $I(S)$ is the mass moment of inertia per unit reference length. The final integral is the rotatory inertia term and is discussed below. We assume that $M_{2}=M\left(S_{2}, t\right)$, where $M(S, t) \mathbf{k}$ is the moment exerted by the material in $\left(S, S_{2}\right]$ on that in $\left[S_{1}, S\right]$. This is the value at $(S, t)$ of constitutive function $(15)_{2}$. By invoking appropriate boundedness and continuity assumptions, we obtain $M\left(S_{1}, t\right)+M_{1}=0$, and with our previous results the leading terms on the left-hand side of Eq. (24) may then be combined into the expression

$$
\mathbf{k} \int_{S_{1}}^{S_{2}} M^{\prime} \mathrm{d} S+[\mathbf{r} \times \mathbf{F}]_{S_{1}}^{S_{2}} .
$$


In Eq. (25), the second term may be written as the integral of $\mathbf{r}^{\prime} \times \mathbf{F}+\mathbf{r} \times \mathbf{F}^{\prime}$ in which $\mathbf{r}^{\prime}$ and $\mathbf{F}^{\prime}$ are replaced by Eqs. (8) and (23), respectively, and $\mathbf{F}$ is given by Eq. (16). The resulting form of the global balance law is equivalent to the local equation

$$
M^{\prime}+\lambda G=I \theta_{, t},
$$

which effectively determines the otherwise arbitrary function $G(S, t)$ in terms of the motion.

Thus, the equations governing the motion of the film are Eqs. (23) and (26), which are to be taken in conjunction with Eqs. (15) and (16). Essentially, Eq. (26) provides an expression for $G$ while Eq. (23) couples the film behaviour with that of the bulk material and can be regarded as a boundary condition for the solution of Eq. (19) with Eqs. (2), (12) and (13) in $\Omega$.

We close this subsection with some remarks on the form of the rotatory inertia term in Eq. (24). This form is essentially that proposed by Tadjbakhsh (1966) in the context of a theory for the planar motions of an extensible rod, which also serves as a model for the present study of plane motions of a thin film (or plate). Tadjbakhsh regarded the rod as a one-dimensional continuum and specified a constitutive framework for the total mechanical energy density consisting of kinetic energy and strain energy jointly. The model equations were then obtained by a formal application of Hamilton's principle. The constitutive hypotheses for the energy density were tailored a posteriori so that the resulting model had the same structure as in the elementary dynamical theory of rods. Precisely the same form of the theory, again for plane motions of extensible rods, was discussed by Antman (1995, Chapter 4), who restricted the actual two-dimensional motion of a thin body in accordance with the Bernoulli-Euler hypothesis. In these developments, as in this work, the inertia coefficient $I$ is independent of the motion.

An alternative viewpoint in which the inertia coefficients are taken to depend on the strain was advanced recently by Hilgers and Pipkin (1997) and Hilgers (1997). The associated equations of motion, again obtained from Hamilton's principle, involve the strain-dependent part of the inertia in the form of a distributed couple. A similar set of dynamical equations may be obtained by specializing the Cosserat theory of directed surfaces so that the director field is constrained to be aligned with the surface normal but permitted to change length, this being intended to account for the thinning or thickening that accompanies surface strain (Naghdi, 1982, Section 6). In yet another treatment of the dynamics of cylindrical motions of shells, Libai and Simmonds (1998, Chapter 4) require the exact expression for the through-thickness integral of the moment of momentum of a two-dimensional body to be expressible in the form $I \omega$, where $I$ is the (fixed) mass moment of inertia as in the present theory and $\omega$ is a spin that depends on the actual position and velocity fields in the considered thin body. This spin is not in general equal to $\theta_{, t}$. In the associated mechanical power identity the moment on the cross-section is power-conjugate to an angle $\beta$ obtained by integrating $\omega$ with respect to time. While such a formulation has the advantage of delivering an exact consequence of the moment-of-momentum balance for the thin body, it suffers from the drawback that the angle $\beta$ cannot be related to the geometry of the curve used to model the body in the absence of information about the actual position and velocity fields, information that is usually unavailable. 
Regardless of the particular formulation chosen, there remains an open question as to the relationship, if any, of the dynamics predicted by such theories to those predicted by conventional non-linear elasticity theory. Comparisons made in the context of classical linear theory without initial stress or rotatory inertia (see, for example, Bogy and Gracewski, 1983) indicate that solutions of the present model are in accord with those of elasticity theory if the characteristic wavelength of the motion greatly exceeds the film thickness. Thus, we expect our results to be quantitatively meaningful in this limit, even if the rotatory inertia is suppressed. Moreover, the problems we consider are based on equations of motion linearized about a fixed configuration in which the associated rotatory inertia term involves a constant mass moment of inertia, equal to $I$ in the present formulation or a function of the underlying stretch of the film in the case of a strain-dependent inertia coefficient, the difference being equivalent to an adjustment of the film thickness that has no effect on the qualitative behaviour of the solutions.

\subsection{Mechanical power and energy flux}

The equations derived in Section 2.3 lead to a mechanical power balance which in turn may be used to obtain expressions for the fluxes of energy in the film and substrate. This is constructed by scalar multiplying Eq. (23) by $\mathbf{r}_{, t}$, Eq. (26) by $\theta_{, t}$ and integrating the resulting equalities over a part, say $Q$, of the film. To this expression we add the integral of the scalar product of Eq. (19) with $\mathbf{x}_{, t}$ over an arbitrary simply connected region $\Omega$ of the substrate, and then use Green's theorem and integration by parts to reduce the result to

$$
\begin{aligned}
& {\left[\mathbf{F} \cdot \mathbf{r}_{, t}\right]_{\partial Q}+\left[M \theta_{, t}\right]_{\partial Q}-\int_{\partial \Omega} \mathbf{E} \cdot \mathbf{N} \mathrm{d} S} \\
& \quad=\int_{\Omega}\left[K_{, t}^{\mathrm{S}}+\operatorname{tr}\left(\mathbf{S} \mathbf{A}_{, t}\right)\right] \mathrm{d} A+\int_{Q}\left(K_{, t}^{\mathrm{F}}+\mathbf{F} \cdot \mathbf{r}_{, t}^{\prime}+M \theta_{, t}^{\prime}-\lambda G \theta_{, t}+\mathbf{S r}_{, t} \cdot \mathbf{N}\right) \mathrm{d} S .
\end{aligned}
$$

Here, the notation $[\cdot]_{\partial Q}$ is used to denote the difference between the values of the enclosed quantity at the endpoints of $Q$, while $K^{\mathrm{S}}$ and $K^{\mathrm{F}}$, respectively are the kinetic energy densities of the substrate and film, defined by

$$
2 K^{\mathrm{S}}=\rho\left|\mathbf{x}_{, t}\right|^{2}, \quad 2 K^{\mathrm{F}}=\rho_{0}\left|\mathbf{r}_{, t}\right|^{2}+I\left(\theta_{, t}\right)^{2},
$$

and

$$
\mathbf{E}=-\mathbf{S} \mathbf{x}_{, t}
$$

is the energy flux in the substrate. To the second integrand on the right-hand side of Eq. (27) we apply Eq. (16) and obtain

$$
\mathbf{F} \cdot \mathbf{r}_{, t}^{\prime}+M \theta_{, t}^{\prime}-\lambda G \theta_{, t}=F \tau \cdot \mathbf{r}_{, t}^{\prime}+G\left(\boldsymbol{v} \cdot \mathbf{r}_{, t}^{\prime}-\lambda \theta_{, t}\right)+M \theta_{, t}^{\prime},
$$

wherein the term in parentheses vanishes identically by virtue of the relation

$$
\mathbf{r}_{, t}^{\prime}=\lambda_{, t} \tau+\lambda \theta_{, t} \boldsymbol{v}
$$

which follows by differentiating Eq. (8). Then, on recalling Eqs. (11) and (15), we see that the right-hand side of Eq. (30) reduces to $F \lambda_{, t}+M \theta_{, t}^{\prime}=U_{, t}$. We also have 
$\operatorname{tr}\left(\mathbf{S A}_{, t}\right)=W_{, t}$, and, if $\Omega$ is chosen such that $Q \subset \partial \Omega$ we may use $\left.\mathbf{x}_{, t}\right|_{Q}=\mathbf{r}_{, t}$ to write Eq. (27) in the form

$$
\left[\mathbf{F} \cdot \mathbf{r}_{, t}\right]_{\partial Q}+\left[M \theta_{, t}\right]_{\partial Q}-\int_{\partial \Omega \backslash Q} \mathbf{E} \cdot \mathbf{N} \mathrm{d} S=\int_{\Omega} \mathscr{E}_{, t} \mathrm{~S} \mathrm{~d} A+\int_{Q} \mathscr{E}_{, t}^{\mathrm{F}} \mathrm{d} S,
$$

where

$$
\mathscr{E}^{\mathrm{S}}=W+K^{\mathrm{S}}, \quad \mathscr{E}^{\mathrm{F}}=U+K^{\mathrm{F}}
$$

are the mechanical energy densities of the substrate and film, respectively. This is the mechanical power identity for coupled film/substrate motions.

The conventional power identity for the substrate material follows from Eq. (19) by taking the region $\Omega$ to be contained entirely within its interior. This yields

$$
\int_{\Omega} \mathscr{E}_{, t} \mathrm{~S} \mathrm{~d} A+\int_{\partial \Omega} \mathbf{E} \cdot \mathbf{N} \mathrm{d} S=0
$$

which is equivalent to the local conservation law

$$
\mathscr{E}_{, t}^{\mathrm{S}}+\operatorname{Div} \mathbf{E}=0 .
$$

A similar balance law for the film may be derived directly from the differential equations that hold on $Q$, or, alternatively, by using Eq. (35) in Eq. (32) to obtain

$$
\int_{Q} \mathscr{E}_{, t}{ }_{F}^{\mathrm{F}} \mathrm{d} S=\int_{Q} \mathbf{E} \cdot \mathbf{N} \mathrm{d} S+\left[\mathbf{F} \cdot \mathbf{r}_{, t}\right]_{\partial Q}+\left[M \theta_{, t}\right]_{\partial Q} .
$$

This in turn is equivalent to the local equation

$$
\mathscr{E}_{, t} \mathrm{~F}+E^{\prime}=\mathbf{E} \cdot \mathbf{N},
$$

where

$$
E=-\mathbf{F} \cdot \mathbf{r}_{, t}-M \theta_{, t}
$$

is the energy flux in the film. From Eq. (37) it is evident that the substrate supplies energy to the film through the flux $\mathbf{E} \cdot \mathbf{N}$.

\section{Incremental motions}

Henceforth, we shall be concerned with a small incremental motion superimposed on an initial quasi-static finite deformation. The equations governing this initial deformation are given by Eqs. (2), (12), (13) and (19) for the bulk material with the time dependence omitted and by Eqs. (15), (16), (25) and (26) for the coating. Let a superimposed dot denote an increment in the quantity concerned. In particular, $\dot{\mathbf{x}}, \dot{\mathbf{S}}, \dot{p}$ are increments in $\mathbf{x}, \mathbf{S}$ and $p$, respectively. The incremental version of the equation of motion (19) is then

$\operatorname{Div} \dot{\mathbf{S}}=\rho \dot{\mathbf{x}}_{, t}$.

Let $\mathbf{u}(\mathbf{x}, t)$ denote the incremental displacement, identified with $\dot{\mathbf{x}}(\mathbf{X}, t)$ through the change of variable $\mathbf{x}=\chi(\mathbf{X})$, which defines the initial finite deformation. With $(\mathbf{x}, t)$ 
regarded as independent variables, Eq. (39) may be expressed in the form

$$
\operatorname{div} \Sigma=\rho \mathbf{u}_{, t t},
$$

where div is the divergence operator in $\hat{\Omega}$ and $\boldsymbol{\Sigma}=\mathbf{A} \dot{\mathbf{S}}$.

The (linearized) incremental form of the constitutive law (13) may be written as

$$
\Sigma=\mathscr{A}_{0} \boldsymbol{\Gamma}+p \boldsymbol{\Gamma}-\dot{p} \mathbf{I},
$$

where $\mathbf{I}$ is the (two-dimensional) identity tensor, $\boldsymbol{\Gamma}=\operatorname{grad} \mathbf{u}$, with grad being the gradient operator in $\hat{\Omega}$, is the displacement gradient having components

$$
\Gamma_{i j}=u_{i, j} \equiv \frac{\partial u_{i}}{\partial x_{j}}
$$

and $\mathscr{A}_{0}$ is the (fourth-order) tensor of instantaneous elastic moduli. Explicit expressions for the components of $\mathscr{A}_{0}$ are given in, for example, Ogden (1984) and are not listed here. Note, however, that $\mathscr{A}_{0}$ and $p$ depend on the form of the initial finite deformation.

The incremental form of incompressibility condition (12), duly linearized, is

$$
\operatorname{div} \mathbf{u}=0 .
$$

On taking the increments of Eqs. (22) and (24) and updating from the variable $S$ to $s$, noting that $\lambda=\mathrm{d} s / \mathrm{d} S$, we obtain

$$
\dot{\mathbf{F}}^{\prime}(s, t)=\Sigma^{\mathrm{T}} \mathbf{n}+\lambda^{-1} \rho_{0} \mathbf{w}_{, t}(s, t)
$$

and

$$
\dot{M}^{\prime}(s, t)+\lambda^{-1} \dot{\lambda} G+\dot{G}=\lambda^{-1} I \dot{\theta}_{, t t}(s, t),
$$

where the prime now signifies partial differentiation with respect to $s, \mathbf{w}=\mathbf{u}$ for $\mathbf{u}$ evaluated on $\hat{P}$, and $\mathbf{n}$ is the rightward unit normal to $\partial \hat{\Omega}$, use having been made of Nanson's formula in the form $\lambda \mathbf{A}^{\mathrm{T}} \mathbf{n}=\mathbf{N}$. It is straightforward to verify that the form of the inertia term in the last equation is appropriate whether or not $I$ is strain dependent due to the fact that the linearization is carried out with respect to a fixed configuration of the film.

In Eqs. (44) and (45) we require the incremental counterparts of Eqs. (15) and (16), namely

$$
\dot{F}=U_{\lambda \lambda} \dot{\lambda}+U_{\lambda \kappa} \dot{\kappa}, \quad \dot{M}=U_{\lambda \kappa} \dot{\lambda}+U_{\kappa \kappa} \dot{\kappa}
$$

and

$$
\dot{\mathbf{F}}=\dot{F} \tau+\dot{G} \boldsymbol{v}+F \dot{\tau}+G \dot{v},
$$

which, in turn, require the expressions

$$
\begin{aligned}
& \lambda^{-1} \dot{\lambda}=\tau \cdot \mathbf{w}^{\prime}, \quad \dot{\kappa}=\lambda \boldsymbol{v} \cdot \mathbf{w}^{\prime \prime}-\kappa \tau \cdot \mathbf{w}^{\prime}, \\
& \dot{\tau}=\left(\boldsymbol{v} \cdot \mathbf{w}^{\prime}\right) \boldsymbol{v}, \quad \dot{\boldsymbol{v}}=-\left(\boldsymbol{v} \cdot \mathbf{w}^{\prime}\right) \tau,
\end{aligned}
$$

these being derived by making use of the incremental versions of Eqs. (8), (10) and (11), as described in (I). We also note that

$$
\dot{\theta}=\boldsymbol{v} \cdot \mathbf{w}^{\prime}, \quad \mathbf{w}^{\prime}=\lambda^{-1} \dot{\lambda} \tau+\left(\boldsymbol{v} \cdot \mathbf{w}^{\prime}\right) \boldsymbol{v} .
$$




\section{Application to a coated half-plane}

We now consider a half-plane subject to a pure homogeneous strain with stretches $\lambda_{1}, \lambda_{2}$ satisfying Eq. (6), and we take the coordinate axes to coincide with the principal axes of strain. In the deformed configuration the half-plane corresponds to $x_{2}<0$ and the surface coating is on the boundary $x_{2}=0$. The coating stretch $\lambda$ is then equal to the stretch $\lambda_{1}$, and hence, by Eq. (6), we have $\lambda_{2}=\lambda^{-1}$.

We may therefore regard $W$ as a function of $\lambda$ defined through

$$
\hat{W}(\lambda)=W\left(\lambda, \lambda^{-1}\right)
$$

from which, with the help of Eq. (14), we deduce that

$$
\sigma_{1}-\sigma_{2}=\lambda \hat{W}^{\prime}(\lambda)
$$

For the superimposed incremental motion the incompressibility condition (43) enables the components $u_{1}, u_{2}$ of the displacement to be expressed in the form

$$
u_{1}=\psi_{, 2}, \quad u_{2}=-\psi_{1},
$$

where $\psi\left(x_{1}, x_{2}, t\right)$ is a scalar function.

Following, for example, Dowaikh and Ogden (1990) we may use Eq. (53) together with Eqs. (41) and (42) in Eq. (40) to eliminate $\dot{p}$ and obtain an equation for $\psi$ for the region $x_{2}<0$. This is

$$
\alpha \psi_{, 1111}+2 \beta \psi_{1122}+\gamma \psi_{, 2222}=\rho\left(\psi_{111 t}+\psi_{, 22 t t}\right),
$$

where the material parameters $\alpha, \beta, \gamma$ (constants here) are given by

$$
\alpha=\lambda^{4} \gamma=\frac{\lambda^{5} \hat{W}^{\prime}(\lambda)}{\lambda^{4}-1}, \quad 2 \beta+2 \gamma=\lambda^{2} \hat{W}^{\prime \prime}(\lambda)
$$

We note that the strong ellipticity inequalities in this case require

$$
\alpha>0, \quad \beta>-\gamma \lambda^{2},
$$

as in Ogden (1984), for example. Since, in its deformed configuration, the film on $x_{2}=0$ is straight, we have $\kappa=0$. Also, $U(\lambda, \kappa)$ is constant with respect to arclength so that $M^{\prime}=0$, and, from Eq. (26) specialized to the static case, we have $G=0$, while $\mathbf{F}=U_{\lambda}(\lambda, 0) \tau$ with $\tau=-\mathbf{e}_{1}$, and $\boldsymbol{v}=-\mathbf{e}_{2}$. From Eq. (23) we also deduce that $\sigma_{2}=0$, so that Eq. (52) is modified accordingly. We note, however, that Eq. (23) may be modified to accommodate non-zero $\sigma_{2}$ if required.

From Eqs. (46) and (47) we then have

$$
\dot{\mathbf{F}}=\left(U_{\lambda \lambda} \dot{\lambda}+U_{\lambda \kappa} \dot{\kappa}\right) \tau+U_{\lambda} \dot{\tau}+\dot{G} \boldsymbol{v},
$$

with the derivatives of $U$ evaluated for $\kappa=0$, together with

$$
\dot{\lambda}=-\lambda w_{1}^{\prime}, \quad \dot{\kappa}=-\lambda w_{2}^{\prime \prime}, \quad \dot{\tau}=w_{2}^{\prime} \mathbf{e}_{2},
$$

and hence

$$
\dot{\mathbf{F}}=\lambda\left(U_{\lambda \lambda} w_{1}^{\prime}+U_{\lambda \kappa} w_{2}^{\prime \prime}\right) \mathbf{e}_{1}+\left(U_{\lambda} w_{2}^{\prime}-\dot{G}\right) \mathbf{e}_{2} .
$$


Eq. (44) now yields the two equations

$$
\begin{aligned}
& \lambda U_{\lambda \lambda} w_{1}^{\prime \prime}+\lambda U_{\lambda \kappa} w_{2}^{\prime \prime \prime}=\Sigma_{21}+\lambda^{-1} \rho_{0} w_{1, t t}, \\
& U_{\lambda} w_{2}^{\prime \prime}-\dot{G}^{\prime}=\Sigma_{22}+\lambda^{-1} \rho_{0} w_{2, t t},
\end{aligned}
$$

while, from Eq. (45), with the help of Eqs. $(46)_{2},(58)$ and (50), we obtain

$$
\dot{G}^{\prime}=\lambda U_{\lambda \kappa} w_{1}^{\prime \prime \prime}+\lambda U_{\kappa \kappa} w_{2}^{\prime \prime \prime \prime}-\lambda^{-1} I w_{2, t}^{\prime \prime},
$$

$I$ being a constant. Substitution of this into Eq. (61) then yields

$$
U_{\lambda} w_{2}^{\prime \prime}-\lambda U_{\lambda \kappa} w_{1}^{\prime \prime \prime}-\lambda U_{\kappa \kappa} w_{2}^{\prime \prime \prime \prime}=\Sigma_{22}+\lambda^{-1} \rho_{0} w_{2, t t}-\lambda^{-1} I w_{2, t t}^{\prime \prime} .
$$

Note that Eq. (60), appropriately specialized and without the $U_{\lambda \kappa}$ term, is similar to an equation used by Murdoch (1976) in the context of waves propagating on a linearly isotropic elastic half-space with a residual surface tension. Eq. (62), on the other hand, has no counterpart in the Murdoch theory. In isolation from the half-plane, Eq. (62) is the equation of motion of a thin rod accounting for rotatory inertia (Antman, 1995). In the theory describing waves propagating in a thin layer on a half-space in the classical context it is sometimes assumed that the rotatory inertia term (involving $I$ ) is negligible compared with the term in $\rho_{0}$, at least in the low-frequency regime (see, for example, Tiersten, 1969). As we show in Section 5, however, it is in general necessary to include the term in $I$. Without the $I$ term and with $U_{\lambda}=U_{\lambda \kappa}=0$, a version of Eq. (62) appropriate for the classical theory was given in Tiersten (1969), as was the counterpart of Eq. (60).

From the specialization of Eq. (41) to the present circumstances (in which $\sigma_{2}=0$ ), we have

$$
\Sigma_{21}=\gamma\left(\psi_{, 22}-\psi_{11}\right),
$$

and, after differentiation of $\Sigma_{22}$ with respect to $x_{1}$ followed by use of the first component of Eq. (40) to eliminate $\dot{p}$,

$$
-\Sigma_{22,1}=(2 \beta+\gamma) \psi_{, 112}+\gamma \psi_{, 222}-\rho \psi_{, 2 t t} .
$$

Details of the derivation of Eq. (64) may be found in, for example, Dowaikh and Ogden (1990).

By taking $s=-x_{1}$ we may also write

$$
w_{1}(s, t)=\psi_{2}\left(x_{1}, 0, t\right), \quad w_{2}(s, t)=-\psi_{, 1}\left(x_{1}, 0, t\right),
$$

so that Eqs. (60) and (62) may be cast in terms of $\psi$ to give

$$
\lambda U_{\lambda \kappa} \psi_{, 1111}+\lambda U_{\lambda \lambda} \psi_{, 112}-\rho_{0} \lambda^{-1} \psi_{, 2 t t}+\gamma \psi_{, 11}-\gamma \psi_{, 22}=0
$$

and

$$
\begin{aligned}
& \lambda U_{\kappa \kappa} \psi_{, 111111}+\lambda U_{\lambda \kappa} \psi_{, 11112}-U_{\lambda} \psi_{, 1111}-\lambda^{-1} I \psi_{, 1111 t t} \\
& \quad+\rho_{0} \lambda^{-1} \psi_{, 11 t t}+(2 \beta+\gamma) \psi_{, 112}+\gamma \psi_{, 222}-\rho \psi_{, 2 t t}=0,
\end{aligned}
$$

respectively, each of which is evaluated on $x_{2}=0$ and for $\kappa=0$. Eqs. (66) and (67) provide boundary conditions on $x_{2}=0$ for the solution $\psi$ of Eq. (54). 


\subsection{Surface waves}

Surface waves on a pre-stressed half-space of incompressible isotropic elastic material without a surface coating were examined by Dowaikh and Ogden (1990). Here we evaluate the effect that the surface coating has on such waves.

We seek solutions of the form

$$
\psi=A \mathrm{e}^{\mathrm{i} k\left(x_{1}-c t\right)+s k x_{2}},
$$

where $c(>0)$ is the wave speed, $k(>0)$ is the wave number while $s$ is to have positive real part so that $\psi \rightarrow 0$ as $x_{2} \rightarrow-\infty$ and is determined by substitution of Eq. (68) into Eq. (54). We distinguish between this $s$ and the arclength parameter $s$ used for $\hat{P}$ used earlier, which does not feature in the remainder of the paper. Let $s_{1}, s_{2}$ be the two solutions with positive real part. Then, the general solution of Eq. (54) of the given form with the desired properties is written as

$$
\psi=\left(A \mathrm{e}^{s_{1} k x_{2}}+B \mathrm{e}^{s_{2} k x_{2}}\right) \mathrm{e}^{\mathrm{i} k\left(x_{1}-c t\right)},
$$

as in Dowaikh and Ogden (1990), and $s_{1}, s_{2}$ are such that

$$
s_{1}^{2}+s_{2}^{2}=\left(2 \beta-\rho c^{2}\right) / \gamma, \quad s_{1}^{2} s_{2}^{2}=\left(\alpha-\rho c^{2}\right) / \gamma .
$$

We recall from Dowaikh and Ogden (1990) that

$$
0 \leqslant \rho c^{2} \leqslant \alpha,
$$

but, as discussed in Ogden and Sotiropoulos (1995), the upper limit $\alpha$ is replaced by a lower limit if $2 \beta<\alpha$ in order to ensure that the decay condition is satisfied (this is made explicit below).

Substitution of Eq. (69) into boundary conditions Eq. (66) and Eq. (67) for $x_{2}=0$ yields

$$
\begin{aligned}
& \left(a+b s_{1}-s_{1}^{2}\right) A+\left(a+b s_{2}-s_{2}^{2}\right) B=0, \\
& \left(u+v s_{1}-s_{1}^{3}\right) A+\left(u+v s_{2}-s_{2}^{3}\right) B=0,
\end{aligned}
$$

where the parameters $a, b, u, v$ are defined by

$$
\begin{aligned}
& a=\left(k^{2} \lambda U_{\lambda \kappa}-\gamma\right) / \gamma, \quad b=k\left(\lambda^{-1} \rho_{0} c^{2}-\lambda U_{\lambda \lambda}\right) / \gamma, \quad v=\left(2 \beta-\gamma a-\rho c^{2}\right) / \gamma, \\
& u=\left(\lambda k^{3} U_{\kappa \kappa}+k U_{\lambda}-\lambda^{-1} I k^{3} c^{2}-k \lambda^{-1} \rho_{0} c^{2}\right) / \gamma .
\end{aligned}
$$

Note that if Eq. (23) is modified to allow for a normal stress on the boundary $x_{2}=0$ then $\sigma_{2} \neq 0$ and $\sigma_{2}$ enters the coefficients (73) only through the parameter $a$ and as an additional term $\sigma_{2} / \gamma$ in $a$. Thus, the effect of such a normal stress is similar to the effect of the term $k^{2} U_{\lambda \kappa}$ in $a$ and could therefore be accommodated by choosing $U$ so that $U_{\lambda \kappa}$ does not vanish.

For a non-trivial solution for $(A, B)$ to exist the determinant of coefficients in Eq. (72) must vanish. After removal of the factor $\left(s_{1}-s_{2}\right)$, whose vanishing is accounted for as a special case of the remaining equation, we obtain

$$
b u-a v-u\left(s_{1}+s_{2}\right)+a\left(s_{1}^{2}+s_{2}^{2}+s_{1} s_{2}\right)-v s_{1} s_{2}+b s_{1} s_{2}\left(s_{1}+s_{2}\right)-s_{1}^{2} s_{2}^{2}=0 .
$$


We make Eq. (74) explicit by introducing the notation $\eta$ defined by

$$
\eta=s_{1} s_{2}=\sqrt{\left(\alpha-\rho c^{2}\right) / \gamma}
$$

so that

$$
\rho c^{2}=\alpha-\gamma \eta^{2}
$$

and hence, using Eq. (70),

$$
s_{1}^{2}+s_{2}^{2}=\eta^{2}+(2 \beta-\alpha) / \gamma, \quad s_{1}+s_{2}=\left[\eta^{2}+2 \eta+(2 \beta-\alpha) / \gamma\right]^{1 / 2} .
$$

Corresponding to Eq. (71) we have

$$
0 \leqslant \eta \leqslant \sqrt{\alpha / \gamma}=\lambda^{2}
$$

but if $2 \beta<\alpha$ the left-hand side limit is replaced by $\eta_{\mathrm{L}} \leqslant \eta$, where $\eta_{\mathrm{L}}>0$ is the positive solution of

$$
\eta_{\mathrm{L}}^{2}+2 \eta_{\mathrm{L}}+(2 \beta-\alpha) / \gamma=0
$$

as discussed in Ogden and Sotiropoulos (1995) and Chadwick (1995).

Note that $a$ in Eq. (73) is independent of $c$, while $b, u, v$, when expressed in terms of $\eta$, may be written

$$
b=\bar{b}-k \bar{\rho} \eta^{2}, \quad u=\bar{u}+r \eta^{2}, \quad v=\bar{v}+\eta^{2},
$$

where

$$
\begin{aligned}
& \bar{b}=k\left(\bar{\rho} \alpha-\lambda U_{\lambda \lambda}\right) / \gamma, \quad \bar{u}=\left(k^{3} \lambda U_{\kappa \kappa}+k U_{\lambda}\right) / \gamma-\lambda^{4} r, \\
& r=\lambda^{-1} I k^{3} / \rho+k \bar{\rho}, \quad \bar{v}=(2 \beta-\alpha) / \gamma-a
\end{aligned}
$$

and $\bar{\rho}$ is defined as

$$
\bar{\rho}=\lambda^{-1} \rho_{0} / \rho \text {. }
$$

Except for $k$ and $\bar{\rho}$ the parameters defined above are dimensionless.

Using Eqs. (75) and (77), and the notation defined in Eqs. (80)-(82) we may re-express Eq. (74) as an equation for $\eta$ in the form

$$
\begin{gathered}
\left(k \bar{\rho} \eta^{3}+r \eta^{2}-\bar{b} \eta+\bar{u}\right)\left[\eta^{2}+2 \eta+(2 \beta-\alpha) / \gamma\right]^{1 / 2}+k \bar{\rho} r \eta^{4} \\
+\eta^{3}+(k \bar{\rho} \bar{u}-r \bar{b}+1) \eta^{2}+(\bar{v}-a) \eta-a^{2}-\bar{b} \bar{u}=0 .
\end{gathered}
$$

This is the secular equation which determines $\eta$, and hence the wave speed through Eq. (76), in terms of the various material parameters of the bulk and film material, the deformation $\lambda$ and the wave number $k$. Note that at this point no specialization of $\hat{W}(\lambda)$ or $U(\lambda, \kappa)$ has been adopted.

When there is no surface coating we have $\bar{\rho}=\bar{b}=r=\bar{u}=0$ and $a=-1$, in which case Eq. (83) reduces to the cubic

$$
\eta^{3}+\eta^{2}+(2 \beta-\alpha+2 \gamma) \eta / \gamma-1=0
$$

which is a special case (corresponding to $\sigma_{2}=0$ ) of an equation given by Dowaikh and Ogden (1990). 
Eq. (83) simplifies considerably for materials with $2 \beta=\alpha+\gamma$, which, for the present (plane strain) situation, forces the strain energy to be of the neo-Hookean form, so that

$$
\hat{W}(\lambda)=\frac{1}{2} \mu\left(\lambda^{2}+\lambda^{-2}-2\right),
$$

where $\mu$ is the shear modulus in the natural configuration. Eq. (83) then becomes a quartic for $\eta$, namely

$$
\begin{aligned}
& k \bar{\rho}(r+1) \eta^{4}+(k \bar{\rho}+r+1) \eta^{3}+[k \bar{\rho} \bar{u}-(r+1)(\bar{b}-1)] \eta^{2} \\
& \quad+(\bar{u}-\bar{b}+1-2 a) \eta-\bar{u}(\bar{b}-1)-a^{2}=0,
\end{aligned}
$$

in contrast to cubic equation (84).

For an uncoated half-plane (with $\left.\sigma_{2}=0\right) \eta$ satisfies cubic equation (84) and the material constants enter in the combination $(2 \beta+2 \gamma-\alpha) / \gamma$, which also features in Eq. (83) through the term $\bar{v}-a$. For the coated half-space, by contrast, Eq. (83) is not in general a polynomial for $\eta$, but for materials with $2 \beta=\alpha+\gamma$, in particular, Eq. (86) is a quartic for $\eta$. While for the uncoated case waves are non-dispersive, the dependence of Eqs. (83) and (86) on the wave number $k$ ensures that waves on a coated half-plane are dispersive.

In order to illustrate the effect that the coating has on the propagation of surface waves we focus on Eq. (86), which captures the main features involved. Specifically, in Section 7, we solve Eq. (86) numerically for $\eta$ for given values of the constants and hence obtain the wave speed in the form (for a neo-Hookean material)

$$
\zeta \equiv \rho c^{2} / \mu=\lambda^{2}-\lambda^{-2} \eta^{2},
$$

which, recalling Eq. (76), defines the notation $\zeta$.

\section{Material parameters and non-dimensionalization}

\subsection{Residual stress and moment}

Industrial thin-film deposition processes invariably leave residual stresses and moments in the film. It is therefore appropriate to incorporate both residual stress and moment in the constitutive description of the film material. They can be accounted for by using the quadratic energy function

$$
U=F_{0}(\lambda-1)+M_{0} \kappa+\frac{1}{2} \mu^{\prime}(\lambda-1)^{2}+C \kappa(\lambda-1)+\frac{1}{2} \mu^{\prime \prime} \kappa^{2},
$$

where $\mu^{\prime}, \mu^{\prime \prime}, F_{0}, M_{0}$ and $C$ are constants, and hence

$$
F=F_{0}+\mu^{\prime}(\lambda-1)+C \kappa, \quad M=M_{0}+C(\lambda-1)+\mu^{\prime \prime} \kappa,
$$

with $U_{\lambda \lambda}=\mu^{\prime}, U_{\lambda \kappa}=C, U_{\kappa \kappa}=\mu^{\prime \prime}$. The energy Eq. (88) is a generalization of the simple model used in (I) that did not include the terms in $F_{0}, M_{0}$ and $C$.

For the considered problem $\kappa=0$ and $F_{0}, M_{0}$ respectively are the residual stress and couple in the undeformed configuration. Since Eq. (89) are constants, the undeformed configuration is equilibrated with $\mathbf{S}=\mathbf{0}$ in the bulk material. Note that $M_{0}$ does not 
feature in the incremental equations, but that the moment $M$ in the deformed configuration is detected in these equations through $C$. The term in $C$ is therefore included to reflect moment effects in the incremental equations. In accordance with Eq. (17), we must have

$$
\mu^{\prime} \geqslant 0, \quad \mu^{\prime \prime} \geqslant 0, \quad \mu^{\prime} \mu^{\prime \prime} \geqslant C^{2} .
$$

L.B. Freund has shown us a simple argument, based on a film of finite thickness $h$, which may be used to motivate expression Eq. (88) for the strain-energy function. Let the stretch and curvature of the film at its centre-line be $\bar{\lambda}$ and $\bar{\kappa}$, respectively. Let $\lambda$ and $\kappa$ be the corresponding quantities at the interface between the film and substrate. In general, these will not be equal to $\bar{\lambda}$ and $\bar{\kappa}$. For example, if the film undergoes pure bending, then, with $b=-h / 2$,

$$
\bar{\lambda}-1=\lambda-1+\kappa b, \quad \bar{\kappa}=\kappa,
$$

provided that $|\kappa b| \ll 1$.

Let $\bar{F}$ and $\bar{M}$ be the axial force and bending moment of the film at its centre-line. Then, if $F$ and $M$ are the corresponding axial force and moment acting at the film-substrate interface, we have

$$
F=\bar{F}, \quad M=\bar{M}+\bar{F} b .
$$

Suppose the constitutive equations for the film are $\bar{F}=A(\bar{\lambda}-1)$ and $\bar{M}=B \bar{\kappa}$, where $A$ and $B$ are constants. Then, $F(\lambda, \kappa) \mathrm{d} \lambda+M(\lambda, \kappa) \mathrm{d} \kappa=\mathrm{d} U$, where

$$
U(\lambda, \kappa)=\frac{1}{2} A(\lambda-1)^{2}+A b(\lambda-1) \kappa+\frac{1}{2}\left(B+b^{2} A\right) \kappa^{2}
$$

is the strain-energy function for the interface. This is a special case of Eq. (88) with

$$
\mu^{\prime}=A, \quad \mu^{\prime \prime}=B+b^{2} A, \quad C=A b .
$$

It is noteworthy that Eq. (90) then yields the restrictions

$$
A \geqslant 0, \quad B \geqslant 0
$$

which are independent of $h$. We then have $C=-A h / 2$, which is negative.

Returning to the present model, in (I) it was also noted that the ratio $\left(\mu^{\prime \prime} / \mu^{\prime}\right)^{1 / 2}$ defines a local length scale for the problem, and this was used as a basis for a non-dimensionalization scheme. We now make this explicit by writing this length scale as $\bar{H}$, which is then defined by

$$
\bar{H}=\left(\mu^{\prime \prime} / \mu^{\prime}\right)^{1 / 2} \text {. }
$$

We use this to define dimensionless quantities

$$
\tilde{k}=k \bar{H}, \quad \tilde{\mu}=\mu^{\prime} / \mu \bar{H}=\mu^{\prime \prime} / \mu \bar{H}^{3} .
$$

The length scale $\bar{H}$ is defined through the elastic moduli and is not explicitly a physical dimension which requires measurement. The moduli themselves should be determined from wave propagation experiments based on the theory.

The use of $\bar{H}$, however, enables us to make contact with classical engineering plate theory. Comparison of the appropriate specialization of Eq. (88) with the corresponding 
expression in (plane strain) engineering plate theory, as described, for example, in Timoshenko and Goodier (1951), would suggest the identifications

$$
\mu^{\prime}=E H /\left(1-v^{2}\right), \quad \mu^{\prime \prime}=E H^{3} / 12\left(1-v^{2}\right),
$$

where $E$ and $v$ are, respectively, Young's modulus and Poisson's ratio for a plate and $H$ is the plate thickness. A comparison of Eq. (96) with the same ratio obtained from Eq. (98) then gives $H=2 \sqrt{3} \bar{H}$. It is also useful for defining other physical quantities associated with the coating. For example, we may use the standard connection $E=2 \mu_{\mathrm{c}}(1+v)$, with $\mu_{\mathrm{c}}$ analogous to the shear modulus of the coating material. It then follows that the dimensionless material constant $\tilde{\mu}$, defined in Eq. (97), may be given in the form

$$
\tilde{\mu}=\frac{4 \sqrt{3}}{1-v} \frac{\mu_{\mathrm{c}}}{\mu} .
$$

Thus, the correspondence between our model and engineering plate theory makes explicit the interpretation, discussed in (I), of $\tilde{\mu}$ as a measure of the shear stiffness of the film material relative to that of the bulk material.

In addition to Eq. (97) two further material constants appear in Eq. (86) when specialized in respect of Eq. (88), namely $\bar{\rho}$, defined by Eq. (82), and $\bar{I}$, defined by

$$
\bar{I}=\lambda^{-1} I k^{3} / \rho .
$$

Let $\rho_{0}=\rho_{\mathrm{c}} H$, where $\rho_{\mathrm{c}}$ (again using the plate theory correspondence) is interpreted as the mass (per unit reference area) of the coating. Then

$$
k \bar{\rho}=2 \sqrt{3} \lambda^{-1} \tilde{k} \rho_{\mathrm{c}} / \rho \equiv m \lambda^{-1} \tilde{k},
$$

where we have introduced the notation

$$
m=2 \sqrt{3} \rho_{\mathrm{c}} / \rho .
$$

Now, since $I$ is defined per unit reference length, in plate theory it may be taken to have the form

$$
I=\rho_{0} h^{2} / 12=\rho_{\mathrm{c}} h^{2} H / 12,
$$

obtained from that for a rod of length $h$ about an axis through its centre and perpendicular to its length. Again using the plate theory correspondence it is convenient to use this form of $I$ for the model considered here. Moreover, we assume the film material to be incompressible (so that $v=1 / 2$ ). Then, $h=\lambda^{-1} H$ and hence, using Eqs. (96), $(97)_{1},(100)$ and $(103)$,

$$
\bar{I}=m \tilde{k}^{3} \lambda^{-3} \text {. }
$$

This is consistent with our assumption that $I$ is constant since the underlying deformation is homogeneous (and hence $\lambda$ is constant) and any associated increment in $I$ does not contribute to the linearized equations (it features only in second-order terms). It also avoids the need to treat $I$ as an additional independent parameter. 
Thus, the coefficients in Eq. (86) may now be simplified according to

$$
\begin{aligned}
& a=-1, \quad \bar{b}=(m-\tilde{\mu}) \tilde{k} \lambda^{3}, \quad r=\left(1+\lambda^{-2} \tilde{k}^{2}\right) m \tilde{k} \lambda^{-1}, \\
& \bar{u}=\left(\lambda \tilde{k}^{2}+\lambda-1\right) \tilde{\mu} \tilde{k} \lambda^{2}-\lambda^{4} r .
\end{aligned}
$$

Note that $\bar{I}$ is negligible if and only if $\tilde{k}^{2} \ll 1$, which corresponds to the low-frequency (or long wavelength) limit. As mentioned in Section 4, it is sometimes assumed in the classical theory that $\bar{I}$ can be neglected, so that rotatory inertia is regarded as unimportant compared with the linear inertia and bending stiffness terms (for example, Tiersten, 1969). Clearly, this is not the case in general since bending stiffness (proportional to $\tilde{\mu}$ ) and rotatory inertia (proportional to $m$ ) each contribute a term of order $\tilde{k}^{3}$ to $\bar{u}$. Thus, we retain the rotatory inertia term here and note that the relative importance of the terms depends on the ratio $\tilde{\mu} / m$.

When, however, $\tilde{k}$ is small then it is straightforward to obtain a solution of Eq. (86) for $\eta$ expanded in powers of $\tilde{k}$. The zero-order solution, say $\eta_{0}$, satisfies the equation

$$
\eta_{0}^{3}+\eta_{0}^{2}+3 \eta_{0}-1=0
$$

which is the secular equation for an uncoated half-plane of neo-Hookean material (Dowaikh and Ogden 1990). This is the Rayleigh wave limit, and hence one branch of the solutions of Eq. (86) must pass through the Rayleigh wave speed value at $\tilde{k}=0$. Eq. (106) has a unique positive solution, given approximately by $\eta_{0}=0.2956$, and the associated wave speed is given by setting $\eta=\eta_{0}$ in Eq. (87). The first-order solution for $\eta$ depends on $\lambda, m$ and $\tilde{\mu}$ but it is a lengthy expression and it is not instructive to give it explicitly here.

In respect of Eq. (88) Eq. (86) takes the form

$$
\begin{aligned}
& m \tilde{k}\left(\lambda^{3}+\lambda^{2} m \tilde{k}+m \tilde{k}^{3}\right) \eta^{4}+\lambda\left(\lambda^{3}+2 \lambda^{2} m \tilde{k}+m \tilde{k}^{3}\right) \eta^{3} \\
& +\lambda\left[\lambda^{3}+\left(\lambda^{2}-\lambda^{6}+\tilde{k}^{2}\right) m \tilde{k}+\tilde{\mu} \tilde{k} \lambda^{6}+m \tilde{\mu} \tilde{k}^{2} \lambda^{3}\left(\lambda^{2} \tilde{k}^{2}+\tilde{k}^{2}+2 \lambda^{2}-\lambda\right)\right. \\
& \left.\quad-2 m^{2} \tilde{k}^{2} \lambda^{3}\left(\lambda^{2}+\tilde{k}^{2}\right)+\lambda^{4} \tilde{k}^{2} m \tilde{F}_{0}\right] \eta^{2}+\lambda^{4}\left[\tilde{\mu} \tilde{k} \lambda^{2}\left(\lambda \tilde{k}^{2}+2 \lambda-1\right)\right. \\
& \left.\quad-m \tilde{k} \lambda\left(2 \lambda^{2}+\tilde{k}^{2}\right)+3+\lambda^{2} \tilde{k} \tilde{F}_{0}-2 \lambda^{3} \tilde{k}^{2} \tilde{C}\right] \eta-\left[\tilde{\mu}\left(\lambda \tilde{k}^{2}+\lambda-1\right) \lambda\right. \\
& \left.\quad-m\left(\lambda^{2}+\tilde{k}^{2}\right)+\lambda \tilde{F}_{0}\right]\left[(m-\tilde{\mu}) \tilde{k} \lambda^{3}-1\right] \tilde{k} \lambda^{5}-\lambda^{4}\left(\lambda^{3} \tilde{k}^{2} \tilde{C}-1\right)^{2}=0,
\end{aligned}
$$

where the dimensionless constants $\tilde{F}_{0}$ and $\tilde{C}$ are defined by

$$
\tilde{F}_{0}=F_{0} / \mu \bar{H}, \quad \tilde{C}=C / \mu \bar{H}^{2} .
$$

In Section 7 we shall present numerical solutions of Eq. (107) for $\zeta$ using connection Eq. (87) with $\tilde{k}$ as the independent variable and for specific choices of the parameters $\lambda, m, \tilde{\mu}, \tilde{C}$ and $\tilde{F}_{0}$. Note that it follows from Eqs. (97), (90) and (108) that

$$
|\tilde{C}| \leqslant \tilde{\mu} \text {. }
$$

We recall that for the example considered earlier in this section $\tilde{C}$ is negative, but in general this need not be the case. 
When Eq. (107) is specialized by setting $\lambda=1, \tilde{F}_{0}=0, \tilde{C}=0$ it may be compared with the corresponding equation obtained by Tiersten (1969), although the latter is for a compressible half-space. Tiersten's equation (58) does not include the cubic and quartic terms in $\tilde{k}$ associated with rotatory inertia, but it does include other cubic and quartic powers of $\tilde{k}$ that are associated with bending stiffness. This omission has a significant effect on the results even at relatively small values of $\tilde{k}$.

\subsection{Specialization to a membrane film}

When specialized to the membrane case we have $\mu^{\prime \prime}=0, C=0$ and $I=0$, but $\rho_{0}$ and $\mu^{\prime}$ are taken to be finite and the previous non-dimensionalization based on $\bar{H}$, which now vanishes, is no longer appropriate. This limit corresponds to $\mu_{\mathrm{c}} H$ and $\rho_{\mathrm{c}} H$ tending to non-zero finite values as $H \rightarrow 0$. In terms of the wave number $k, \bar{\rho}$ as defined by Eq. (82), and the notations defined by

$$
\bar{\mu}=\mu^{\prime} / \mu, \quad \bar{F}_{0}=F_{0} / \mu,
$$

Eq. (107) simplifies to

$$
\begin{aligned}
& k \bar{\rho}(1+k \bar{\rho}) \eta^{4}+(1+2 k \bar{\rho}) \eta^{3}+\left[1+\left(1-\lambda^{4}\right) k \bar{\rho}+\lambda^{3} k \bar{\mu}+\lambda^{2}(2 \lambda-1) k^{2} \bar{\mu} \bar{\rho}\right. \\
& \left.-2 \lambda^{4} k^{2} \bar{\rho}^{2}+\lambda^{2} k^{2} \bar{\rho} \bar{F}_{0}\right] \eta^{2}+\left[\lambda^{2}(2 \lambda-1) k \bar{\mu}-2 \lambda^{4} k \bar{\rho}+3+\lambda^{2} k \bar{F}_{0}\right] \eta \\
& -\lambda^{2} k\left[(\lambda-1) \bar{\mu}-\lambda^{2} \bar{\rho}+\bar{F}_{0}\right]\left[\lambda^{3} k(\lambda \bar{\rho}-\bar{\mu})-1\right]-1=0 .
\end{aligned}
$$

In respect of Eq. (111) we seek solutions for $\zeta$ with $k$ (instead of $\tilde{k}$ ) as the independent variable and for chosen values of the reduced number of parameters $\lambda$, with $\bar{\mu}$ and $\bar{F}_{0}$ instead of $\tilde{\mu}$ and $\tilde{F}_{0}$. The parameters $\bar{\rho}, \bar{\mu}, \bar{F}_{0}$ may be made dimensionless by multiplication by $k$ but this makes interpretation of the asymptotic results for large $k$ unclear. Hence we leave them in dimensional form. Note that, in accordance with Eq. (18), we must have

$$
\bar{F}_{0}+\bar{\mu}(\lambda-1) \geqslant 0 .
$$

In the undeformed configuration with $\lambda=1$ Eq. (111) simplifies further to

$$
\begin{aligned}
& k \bar{\rho}(k \bar{\rho}+1) \eta^{4}+(2 k \bar{\rho}+1) \eta^{3}+\left[k^{2} \bar{\rho} \bar{F}_{0}+(k \bar{\rho}+1) k \bar{\mu}-2 k^{2} \bar{\rho}^{2}+1\right] \eta^{2} \\
& \quad+\left(k \bar{F}_{0}+k \bar{\mu}-2 k \bar{\rho}+3\right) \eta+k^{2} \bar{F}_{0} \bar{\mu}-(k \bar{\rho}-1) k \bar{F}_{0} \\
& \quad-k^{2} \bar{\rho} \bar{\mu}+k^{2} \bar{\rho}^{2}-k \bar{\rho}-1=0 .
\end{aligned}
$$

It can be shown that this is equivalent to the incompressible limit of a result obtained by Murdoch (1976) for compressible materials for this special case by identifying $F_{0}, \mu^{\prime}$ and $\eta$, respectively, with the notations $\sigma, \Gamma$ and $S$ used by Murdoch.

\section{Asymptotic results}

As already noted in Section 5 , in the limit $\tilde{k} \rightarrow 0$ there is a unique solution of Eq. (107) corresponding to the Rayleigh wave speed. Away from this limit the character 
of the Rayleigh wave branch and of the other solutions is strongly influenced by the behaviour of Eq. (107) at large $\tilde{k}$. We therefore now study the limiting case $\tilde{k} \rightarrow \infty$, which requires that the coefficient of the highest power of $\tilde{k}$ in Eq. (107) must vanish. This yields a quadratic in $\zeta$ which has solutions given by

$$
2 m \zeta=\left(\lambda^{2}+1\right) \lambda^{2} \tilde{\mu} \pm \lambda^{2}\left[(\lambda-1)^{2} \tilde{\mu}^{2}+4 \lambda^{2} \tilde{C}^{2}\right]^{1 / 2} .
$$

If, with reference to Eqs. (78) and (87), these two solutions both lie in the interval $\left[0, \lambda^{2}\right]$ then it follows that two branches of the solution to Eq. (107) have large wave number (or high-frequency) asymptotes.

The larger solution lies in the required interval if and only if

$$
\lambda^{2} \tilde{\mu} \leqslant m, \quad \tilde{\mu} \leqslant m, \quad \lambda^{2} \tilde{C}^{2} \leqslant\left(\lambda^{2} \tilde{\mu}-m\right)(\tilde{\mu}-m) .
$$

If Eq. (115) holds then the smaller solution is also in the required interval if

$$
|\tilde{C}| \leqslant \tilde{\mu},
$$

which is just Eq. (109). If Eq. (115) holds but Eq. (116) does not then there is only one asymptote. If Eq. (115) does not hold then for a single asymptote we must have, in addition to Eq. (116), either

$$
\left(\lambda^{2}+1\right) \tilde{\mu} \leqslant 2 m,
$$

or

$$
\left(\lambda^{2}+1\right) \tilde{\mu}>2 m, \quad \lambda^{2} \tilde{C}^{2} \geqslant\left(\lambda^{2} \tilde{\mu}-m\right)(\tilde{\mu}-m) .
$$

If Eq. (115) fails and either of Eq. (116) or Eq. (117) fails then there are no asymptotes. Note that inequalities Eq. (115)-(117) are independent of the residual stress $\tilde{F}_{0}$ and, further, depend only on the ratios $\tilde{\mu} / m$ and $\tilde{C} / m$.

For the special case in which $\lambda=1(\tilde{C} \neq 0)$, for example, Eq. (114) reduces to

$$
m \zeta=\tilde{\mu} \pm \tilde{C}
$$

which, by Eq. (109), are both non-negative. There are two asymptotes if

$$
\tilde{\mu}+|\tilde{C}| \leqslant m,
$$

one if

$$
\tilde{\mu}-|\tilde{C}| \leqslant m<\tilde{\mu}+|\tilde{C}|,
$$

and none if

$$
m<\tilde{\mu}-|\tilde{C}| .
$$

For the situation of a finite thickness layer on a half-space large wave number (high-frequency) asymptotes were discussed by Ogden and Sotiropoulos (1995). One asymptote in that case corresponds to the speed of a surface wave in the layer material and the other to an interfacial wave speed between two half-spaces consisting of the two materials. This interpretation is not applicable in the present circumstances.

Further information about the structure of the branches (other than the Rayleigh branch) is determined by considering where they cut the line $\eta=0\left(\zeta=\lambda^{2}\right)$. The value 
of the wave speed at such a point is that of a shear wave speed in the half-space material. By setting $\eta=0$ in Eq. (107) we obtain a quartic for $\tilde{k}$, namely

$$
\begin{aligned}
& {\left[\left(\lambda^{2} \tilde{\mu}-m\right)(\tilde{\mu}-m)-\lambda^{2} \tilde{C}^{2}\right] \lambda^{4} \tilde{k}^{4}+\lambda\left(\lambda^{2} \tilde{\mu}-m\right) \tilde{k}^{3}} \\
& \quad+\lambda^{2}\left[\lambda^{3}(\tilde{\mu}-m)\left\{\tilde{F}_{0}+\tilde{\mu}(\lambda-1)-m \lambda\right\}+2 \lambda \tilde{C}\right] \tilde{k}^{2} \\
& \quad+\lambda^{2}\left[\tilde{F}_{0}+\tilde{\mu}(\lambda-1)-m \lambda\right] \tilde{k}-1=0 .
\end{aligned}
$$

Note that, in contrast to the asymptotic results, this does depend on $\tilde{F}_{0}$.

For completeness we now note the specialization of Eq. (107) associated with bifurcation. This is obtained by setting $\zeta=0\left(\eta=\lambda^{2}\right)$ and again this yields a quartic in $\tilde{k}$, in this case

$$
\begin{aligned}
& \lambda^{6}\left(\tilde{\mu}^{2}-\tilde{C}^{2}\right) \tilde{k}^{4}+\lambda^{3}\left(\lambda^{2}+1\right) \tilde{\mu} \tilde{k}^{3}+\lambda^{3}\left[\lambda^{2}(\lambda-1) \tilde{\mu}^{2}+\lambda^{2} \tilde{\mu} \tilde{F}_{0}-2\left(\lambda^{2}-1\right) \tilde{C}\right] \tilde{k}^{2} \\
& \quad+\lambda^{2}\left(\lambda^{2}+1\right)\left[\left(\lambda^{3}+\lambda-1\right) \tilde{\mu}+\tilde{F}_{0}\right] \tilde{k}+\lambda^{6}+\lambda^{4}+3 \lambda^{2}-1=0 .
\end{aligned}
$$

As noted in Section 4, when $\tilde{F}_{0}$ and $\tilde{C}$ are set to zero Eq. (123) agrees with Eq. (6.57) in (I), although the latter has to be expanded in terms of $\tilde{k}$ to make this explicit.

From Eq. (123) we may deduce immediately that necessary conditions for the underlying configuration to be stable, i.e. for the left-hand side of Eq. (123) to be positive for all $\tilde{k} \geqslant 0$, are

$$
\lambda^{6}+\lambda^{4}+3 \lambda^{2}-1>0, \quad|\tilde{C}|<\tilde{\mu} .
$$

The first of these is the condition for stability of an uncoated half-plane (Dowaikh and Ogden, 1990), while the second (in its non-strict form) appeared in Eq. (116) in connection with the enumeration of asymptotes. General necessary and sufficient conditions may be written down by considering the detailed properties of the left-hand side of Eq. (123) but they are very complicated and it is not instructive to include them here.

As indicated above, the asymptotic behaviour has a strong influence on that at smaller values of $\tilde{k}$. If situations in which bifurcation can occur are discounted, that is if dispersion curves are not allowed to cut the axis $\zeta=0$, then analysis of the properties of Eq. (122) yields the following results: (a) if there are zero or two high-frequency asymptotes then there are either one or three values of $\tilde{k}$ where $\eta=0$; (b) if there is just one asymptote then there are zero or two (or possibly four) points where $\eta=0$. In principle, the latter possibility in (b) may arise since the permissible ranges of parameter values admit coefficients in Eq. (122) with alternating signs. However, for the parameter values for which numerical results have been obtained this possibility has not been encountered. It may be possible to rule it out by more detailed consideration of the properties of the quartic in Eq. (122) but thus far attempts to do this have not proved successful. A selection of the other cases is illustrated in the numerical results shown in Section 7. 
Results for a membrane corresponding to those discussed above may be deduced on noting that the two asymptotic values of $\zeta$ are

$$
\zeta=\lambda \bar{\mu} / \bar{\rho}, \quad \zeta=\left[\bar{F}_{0}+(\lambda-1) \bar{\mu}\right] / \bar{\rho},
$$

the latter depending on the residual stress.

The membrane equation corresponding to Eq. (122) is a quadratic in $k$, namely

$$
\lambda^{5}(\lambda \bar{\rho}-\bar{\mu})\left[(\lambda-1) \bar{\mu}-\lambda^{2} \bar{\rho}+\bar{F}_{0}\right] k^{2}-\lambda^{2}\left[(\lambda-1) \bar{\mu}-\lambda^{2} \bar{\rho}+\bar{F}_{0}\right] k+1=0,
$$

and that corresponding to Eq. (123) is also a quadratic, in this case

$$
\begin{aligned}
& \lambda^{5} \bar{\mu}\left[\bar{F}_{0}+\bar{\mu}(\lambda-1)\right] k^{2}+\lambda^{2}\left(\lambda^{2}+1\right)\left[\bar{F}_{0}+\bar{\mu}\left(\lambda^{3}+\lambda-1\right)\right] k \\
& \quad+\lambda^{6}+\lambda^{4}+3 \lambda^{2}-1=0,
\end{aligned}
$$

which is independent of $\bar{\rho}$.

It follows from Eq. (127) that necessary and sufficient conditions for stability of the underlying configuration with a membrane coating are $(124)_{1}$ together with the strict form of Eq. (112), namely

$$
\bar{F}_{0}+\bar{\mu}(\lambda-1)>0 .
$$

On consideration of Eq. (126), it is easy to show that for a membrane the following two possibilities arise: (a) if zero or two high-frequency asymptotes exist then there is exactly one value of $k$ where $\eta=0$; (b) if there is only one asymptote then there are zero or two points with $\eta=0$.

\section{Numerical results and discussion}

\subsection{Results for $\lambda=1$}

Fig. 1 shows the effect of a positive residual moment in the absence of residual stress, with the values of the parameters given by $\lambda=1, \tilde{\mu}=1$ and, respectively in (a)-(f), $m=0.5,0.9,1,1.5,3,5$. The long-, medium- and short-dashed curves are for $\tilde{C}=0.5,0.8$ and 1.2 , respectively. Note that for $\tilde{C}=1.2$ inequality $(124)_{2}$ required for stability is violated. As expected from the discussion in Section 6, it can be seen that the presence of non-zero $\tilde{C}$ affects the number and disposition of the asymptotes. Results for negative values of $\tilde{C}$, which are not shown here, are very similar to those for positive $\tilde{C}$ although there are some differences of detail, particularly for small values of $m$. Note, however, that for the example constructed in Section 5.1 $\tilde{C}$ is negative. If $\tilde{C}=0$ the effect of varying $\tilde{F}_{0}$ is very similar to the effect of applying a stretch $\lambda$, which is discussed below, so we do not show such results separately.

\subsection{Results for $\lambda \neq 1$}

In order to illustrate the effect of extensional and compressive stretches in the substrate, we now show results, in Figs. 2 and 3 for $\lambda=1.4$ and 0.7 , respectively. In each case the same values of $\tilde{\mu}$ and $m$ as in Fig. 1 are used. In Fig. 2 the upper limit for $\zeta$ 
(a)

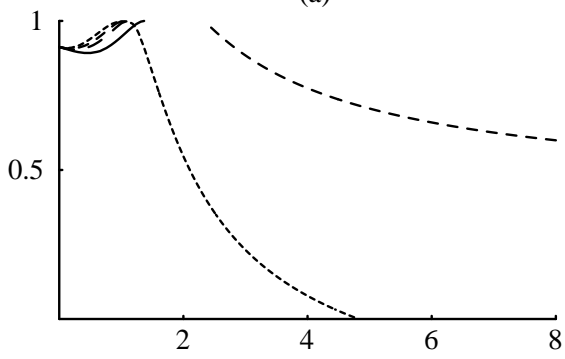

(c)

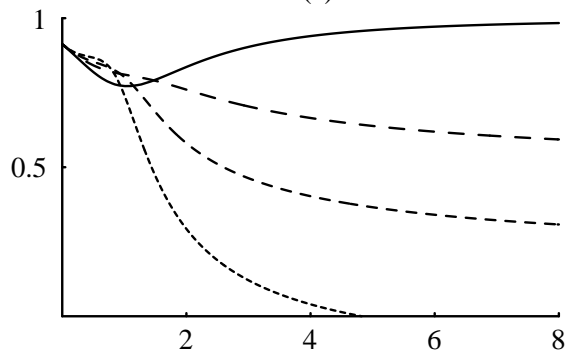

(e)

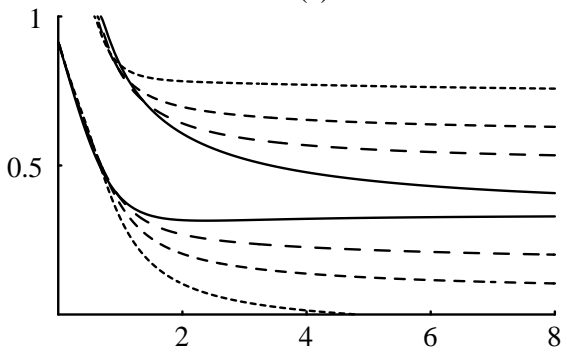

(b)

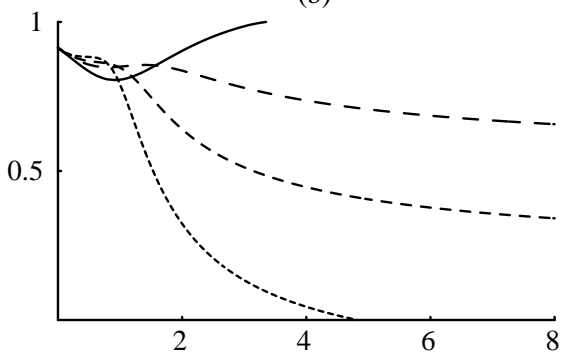

(d)

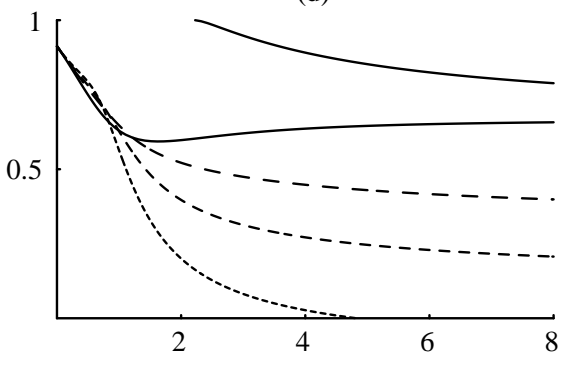

(f)

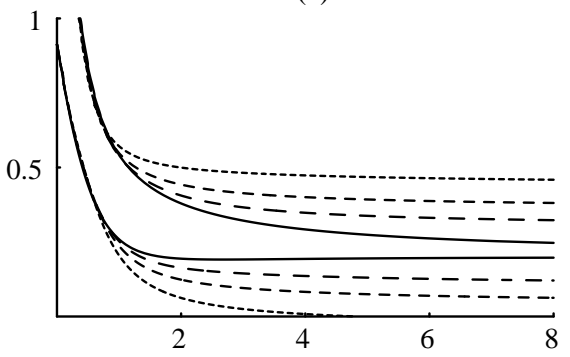

Fig. 1. Plot of $\zeta=\rho c^{2} / \mu$ (vertical scale) against $\tilde{k}$ (horizontal scale) for $\lambda=1, \tilde{\mu}=1, \tilde{F}_{0}=0$ and the following values of $m$ : (a) 0.5 , (b) 0.9 , (c) 1 , (d) 1.5 , (e) 3, (f) 5 . In each figure the continuous (long-dashed, dashed, short-dashed) curves are for $\tilde{C}=0(0.5,0.8,1.2)$.

is 1.96 (compared with 1 in Fig. 1) so that generally a value of $\lambda>1$ has the effect of increasing the wave speed. This is similar to the effect of a tensile residual stress, but results for this are not shown separately. Fig. 2 shows results for positive values of $\tilde{C}$, with $\tilde{F}_{0}=0$. The general character of the results is similar to that in Fig. 1. One value of $|\tilde{C}|$ (i.e. 1.6) has been chosen so that the necessary condition for stability $(124)_{2}$ is violated. Again, results are not shown for negative values of $\tilde{C}$ since they are very similar to those shown in Fig. 2.

In Fig. 3 the upper limit for $\zeta$ is 0.49 and generally the wave speed is reduced by the compressive stretch. With $\tilde{F}_{0}=0$, Fig. 3 shows results for positive values of $\tilde{C}$. The 
(a)

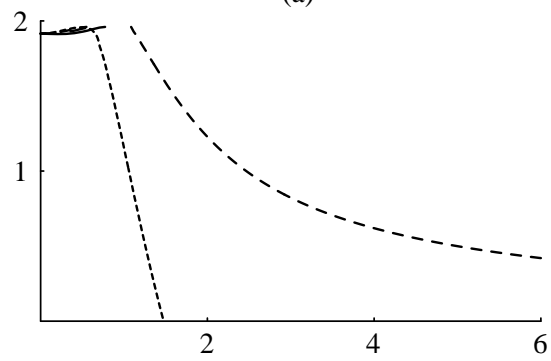

(c)

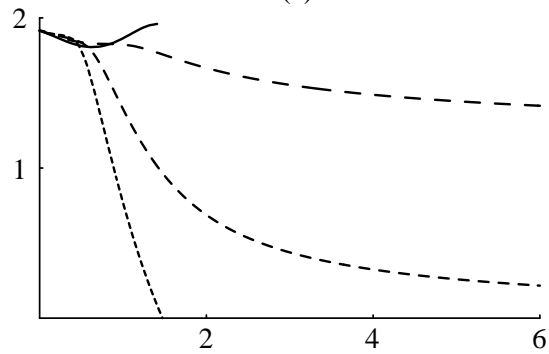

(e)

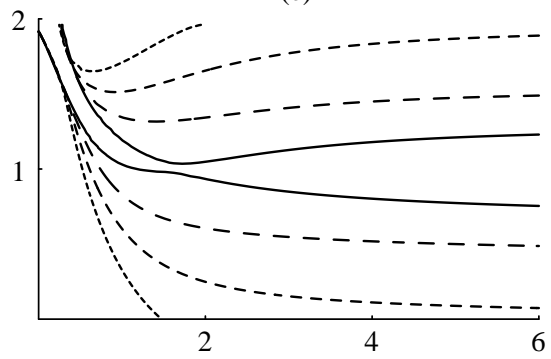

(b)

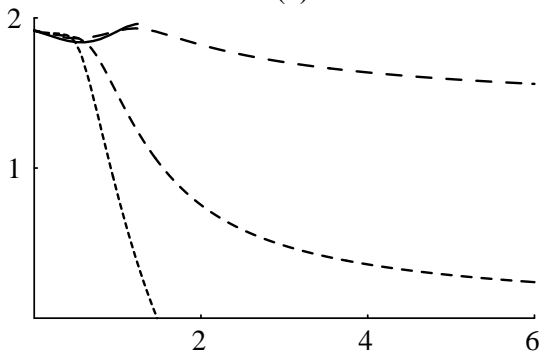

(d)

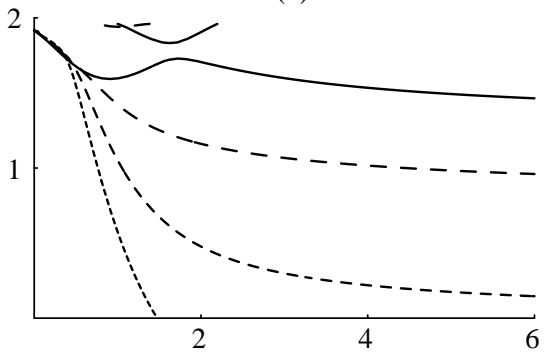

(f)

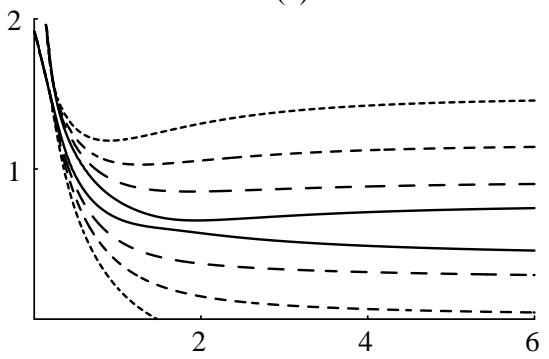

Fig. 2. Plot of $\zeta=\rho c^{2} / \mu$ (vertical scale) against $\tilde{k}$ (horizontal scale) for $\lambda=1.4, \tilde{\mu}=1, \tilde{F}_{0}=0$ and the following values of $m$ : (a) 0.5 , (b) 0.9 , (c) 1, (d) 1.5, (e) 3, (f) 5. In each figure the continuous (long-dashed, dashed, short-dashed) curves are for $\tilde{C}=0(0.5,1,1.6)$.

values of $\tilde{C}$ used are $0.3,0.6$ and 1, corresponding to long-, medium- and short-dashed curves, respectively. The differences between the results for positive and negative values of $\tilde{C}$ are somewhat more marked than for larger stretches, but again we do not show these results here. Calculations not illustrated here also show, as expected, that stability is lost for a smaller value of the compressive residual stress than is the case for $\lambda=1$ or 1.4 .

Calculations have also been carried out for combined non-zero values of $\tilde{F}_{0}$ and $\tilde{C}$, but these do not reveal features that are essentially different from those shown here, so these results are not described separately. Corresponding results for the membrane limit are not included here so as to save space. 
(a)

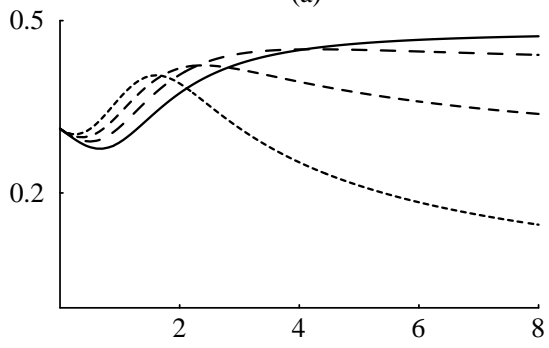

(c)

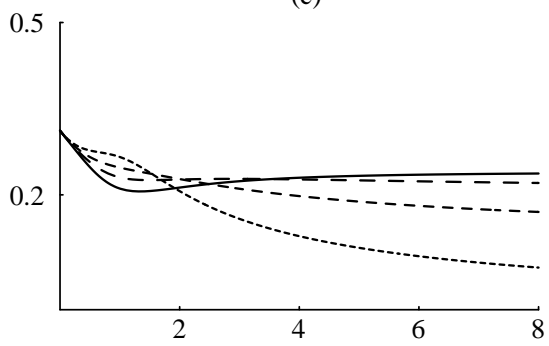

(e)

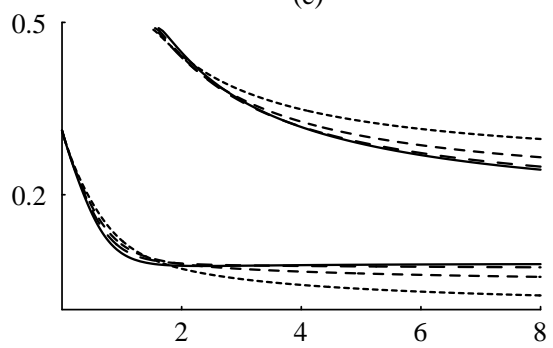

(b)

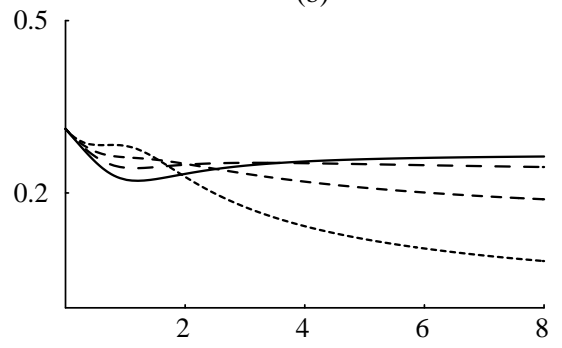

(d)

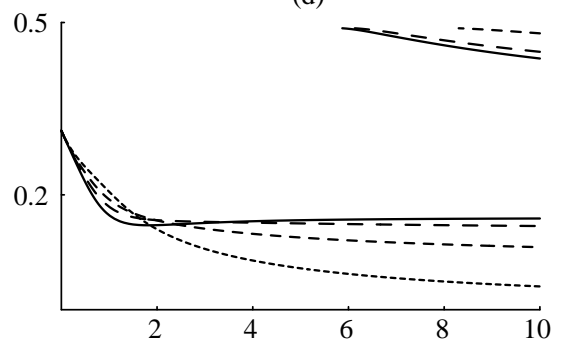

(f)

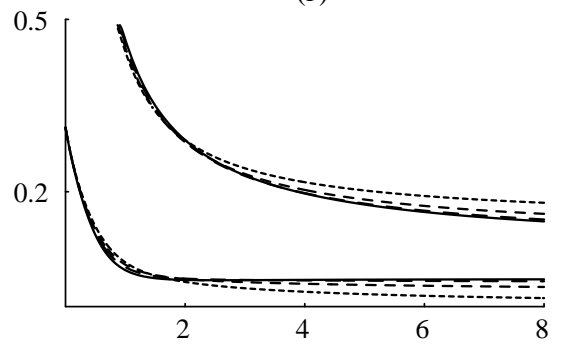

Fig. 3. Plot of $\zeta=\rho c^{2} / \mu$ (vertical scale) against $\tilde{k}$ (horizontal scale) for $\lambda=0.7, \tilde{\mu}=1, \tilde{F}_{0}=0$ and the following values of $m$ : (a) 0.5 , (b) 0.9, (c) 1, (d) 1.5, (e) 3, (f) 5. In each figure the continuous (long-dashed, dashed, short-dashed) curves are for $\tilde{C}=0(0.3,0.6,1)$.

\subsection{Concluding remarks}

The model for a thin film discussed in this paper is an exact (one-dimensional) rod model and the resulting rod equations are exact boundary conditions for the substrate. Unlike classical engineering rod or plate theory, it is not derived by descent from the three-dimensional continuum theory and should be considered on its own merits. Indeed, the films being modelled are too thin to support the continuum hypothesis and our model is therefore designed as an alternative basis for the discussion of thin films. The surface energy $U$ has been taken to depend on just the stretch $\lambda$ and the curvature $\kappa$, but may, more generally, be allowed to depend on higher gradients in order to reflect more structure in the film. 
Classical plate theory is based on various approximations regarding the threedimensional displacement field, and in the version of the theory derived from continuum theory it is known that the conclusions are reliable for $\tilde{k} \ll 1$ (Mindlin, 1951). The nature of the approximations discounts the validity of the theory for larger $\tilde{k}$. By contrast, since the model considered here is exact, there is no a priori physical reason why its validity should be restricted to small $\tilde{k}$. Even if this were not the case, from the mathematical point of view the results for large $\tilde{k}$ would be valuable not least because they dictate the general character of the results for smaller $\tilde{k}$.

\section{Acknowledgements}

This work was supported by a NATO Collaborative Research Grant CRG950152. R.W. Ogden is grateful to the Department of Mechanical Engineering, University of California at Berkeley, for his appointment as Springer Visiting Professor in October 1997.

\section{References}

Achenbach, J.D., Keshava, S.P., 1967. Free waves in a plate supported by a semi-infinite continuum. J. Appl. Mech. 34, 397-404.

Antman, S.S., 1995. Nonlinear Problems of Elasticity. Springer, Berlin.

Bogy, D.B., Gracewski, S.M., 1983. Reflection coefficient for plane waves in a fluid incident on a layered elastic half-space. J. Appl. Mech. 50, 405-414.

Chadwick, P., 1995. Interfacial waves in pre-strained isotropic elastic media. Z. Angew. Math. Phys. 46, S51-S71.

Dowaikh, M.A., Ogden, R.W., 1990. On surface waves and deformations in a pre-stressed incompressible elastic solid. IMA J. Appl. Math. 44, 261-284.

Dryburgh, G., Ogden, R.W., 1999. Bifurcation of an elastic surface-coated incompressible isotropic elastic block subject to bending. Z. Angew. Math. Phys. 50, 822-838.

Farnell, G.W., Adler, E.L., 1972. Elastic wave propagation in thin layers. Phys. Acoust. 9, 35-127.

Gille, G., Rau, R., 1984. Buckling instability and adhesion of carbon layers. Thin Solid Films 120, $109-121$.

Gurtin, M.E., Murdoch, A.I., 1975. A continuum theory of elastic material surfaces. Arch. Rat. Mech. Anal. 57, 291-323.

Gurtin, M.E., Murdoch, A.I., 1979. Surface stress in solids. Int. J. Solids Struct. 14, 431-440.

Hilgers, M.G., 1997. Dynamics of elastic sheets with bending stiffness. Quart. J. Mech. Appl. Math. 50, 525-543.

Hilgers, M.G., Pipkin, A.C., 1997. Kinetic energy of highly elastic membranes. Quart. Appl. Math. 55, 791-800.

Krulevitch, P., Johnson, G.C., Howe, R.T., 1992. Stress and microstructure in LPCVD polycrystalline silicon films: experimental results and closed form modelling of stresses. In: Nix, W.D., Bravman, J.C., Arzt, E., Freund, L.B. (Eds.), Thin films: Stresses and Mechanical Properties, Vol. III. Metals Research Society, Pittsburgh, PA, pp. 13-18.

Kuba, P., Sedláček, V., 1990. Some theoretical problems of shot peening. In: Meguid, S.A. (Ed.), Surface Engineering. Elsevier, London, pp. 393-403.

Libai, A., Simmonds, J.G., 1998. The Nonlinear Theory of Elastic Shells, 2nd Edition. University Press, Cambridge.

Mindlin, R.D., 1951. Influence of rotatory inertia and shear on flexural motions of isotropic, elastic plates. J. Appl. Mech. 18, 31-38. 
Murdoch, A.I., 1976. The propagation of surface waves in bodies with material boundaries. J. Mech. Phys. Solids 24, 137-146.

Naghdi, P.M., 1982. Finite deformation of elastic rods and shells. In: Carlson, D.E., Shield, R.T. (Eds.), Proceedings of the IUTAM Symposium on Finite Elasticity, Lehigh University, 1980. Martinus Nijhoff, The Hague, pp. 47-103.

Nix, W.D., 1989. Mechanical properties of thin films. Metall. Trans. 20A, 2217-2245.

Ogden, R.W., 1984. Non-linear Elastic Deformations. Ellis Horwood, Chichester.

Ogden, R.W., Sotiropoulos, D.A., 1995. On interfacial waves in pre-stressed layered incompressible elastic solids. Proc. Roy. Soc. London A 450, 319-341.

Ogden, R.W., Steigmann, D.J., Haughton, D.M., 1997. The effect of elastic surface coating on the finite deformation and bifurcation of a pressurized circular annulus. J. Elasticity 47, 121-145.

Ogden, R.W., Dryburgh, G., Steigmann, D.J., 1998. The effect of elastic surface coating on the bending of an incompressible elastic block. Z. Angew. Math. Mech. 78 (S1), S145-S148.

Steigmann, D.J., Ogden, R.W., 1997a. Plane deformations of elastic solids with intrinsic boundary elasticity. Proc. Roy. Soc. London A 453, 853-877.

Steigmann, D.J., Ogden, R.W., 1997b. A necessary condition for energy-minimizing plane deformations of elastic solids with intrinsic boundary elasticity. Math. Mech. Solids 2, 3-16.

Steigmann, D.J., Ogden, R.W., 1999. Elastic surface-substrate interactions. Proc. Roy. Soc. London A 455, 437-474.

Tadjbakhsh, I., 1966. The variational theory of the plane motion of the extensible elastica. Int. J. Eng. Sci. 4, 433-450.

Tiersten, H.F., 1969. Elastic surface waves guided by thin films. J. Appl. Phys. 40, 770-789.

Timoshenko, S., Goodier, J.N., 1951. Theory of Elasticity, 2nd Edition. McGraw-Hill, New York.

$\mathrm{Wu}$, C.H., 1996. The chemical potential for stress-driven surface diffusion. J. Mech. Phys. Solids 44, 2059-2077.

Wu, C.H., Hsu, J., Chen, C.-H., 1998. The effect of surface stress on the stability of surfaces of stress solids. Acta Mater. 46, 3755-3760.

Yu, H.W., Kim, C., Sanday, S.C., 1991. Buckle formation in vacuum-deposited thin films. Thin Solid Films 196, 229-233. 